\title{
VLBA Observations of Sub-Parsec Structure in Mrk 231: Interaction between a Relativistic Jet and a BAL Wind
}

\author{
Cormac Reynolds ${ }^{1}$, Brian Punsly ${ }^{2}$, Preeti Kharb ${ }^{3}$ \\ Christopher P. O'Dea ${ }^{3}$ and Joan Wrobel ${ }^{4}$
}

\begin{abstract}
We report on the first high frequency VLBI observations of the nearby broad absorption line quasar (BALQSO), Mrk 231. Three epochs of observations were achieved at $15 \mathrm{GHz}$ and $22 \mathrm{GHz}$, two of these included $43 \mathrm{GHz}$ observations as well. The nuclear radio source is resolved as a compact double. The core component experienced a strong flare in which the flux density at $22 \mathrm{GHz}$ increased by $>150 \%$ (45 mJy) in three months. Theoretical models of the flare imply that the emission is likely enhanced by very strong Doppler boosting of a highly relativistic ejecta with a kinetic energy flux, $Q \sim 3 \times 10^{43} \mathrm{ergs} / \mathrm{sec}$. Combining our data with two previous epochs of $15 \mathrm{GHz}$ data, shows marginal evidence for the slow advance of the secondary component (located $\approx 0.97 \mathrm{pc}$ from the core) over a 9.4 year span. We estimate that the long term time averaged kinetic energy flux of the secondary at $\bar{Q} \approx 10^{42} \mathrm{ergs} / \mathrm{sec}$. Low frequency VLBA observations indicate that the secondary is seen through a shroud of free-free absorbing gas with an emission measure of $\approx 10^{8} \mathrm{~cm}^{-6} \mathrm{pc}$. The steep spectrum secondary component appears to be a compact radio lobe that is associated with a working surface between the ram-pressure confined jet, and a dense medium that is likely to be the source of the free-free absorption. The properties of the dense gas are consistent with the temperatures, displacement from the nucleus and the column density of total hydrogen commonly associated with the BAL wind.
\end{abstract}

Subject headings: quasars: absorption lines — galaxies: active — accretion disks — black holes

\footnotetext{
${ }^{1}$ Curtin University of Technology, Department of Imaging and Applied Physics, GPO Box U1987, Perth, Western Australia, 6102, Australia

${ }^{2} 4014$ Emerald Street No.116, Torrance CA, USA 90503 and ICRANet, Piazza della Repubblica 10 Pescara 65100, Italy, brian.punsly@verizon.net or brian.punsly@comdev-usa.com

${ }^{3}$ Department of Physics, Rochester Institute of Technology, 54 Lomb Memorial Drive,Rochester, NY 14623

${ }^{4}$ NRAO, Socorro, NM 87801-0387 United States
} 


\section{Introduction}

At a redshift of 0.042 , Mrk 231 is one of the nearest radio-quiet quasars to earth. Mrk 231 is a well studied object because it is one of the brightest AGN in the infrared sky. It is a quasar that is partially obscured by dusty gas as evidenced by its red optical/UV spectrum (Lipari et al 1994; Smith et al 1995). The host galaxy has a strong starburst contribution, but the obscured AGN bolometric luminosity, $L_{b o l}$, can be inferred from a pronounced $10 \mu$ peak in the IR spectrum (Farrah et al 2003; Lonsdale et al 2003). Using the extracted IR AGN luminosity, we estimate from QSO composite spectra (see Punsly and Tingav (2005); Telfer et al (2002); Zheng et al (1997)) that the total thermal luminosity created by the central engine is $L_{b o l} \lesssim 2 \times 10^{46} \mathrm{ergs} / \mathrm{s}$ and Mrk 231 would appear as a quasar with $M_{V} \approx-25.3$ if not for the semi-transparent dusty shroud (in agreement with the estimate in Bokensberg et al (1977) 1 . This equates to about 2.7 magnitudes of visual extinction created by the dusty enveloping gas. Comparing the composite spectrum to the optical/UV spectrum in Smith et al (1995) indicates even more severe reddening in the blue band, 3.1 magnitudes at $4400 \AA$. It is the closest radio quiet quasar in which there is sufficient radio flux to permit high signal to noise imaging with high frequency VLBI (Very Long Baseline Interferometry) observations. As a result of the ability to utilize the high resolution of VLBI, this nucleus can be resolved with higher precision than any other radio quiet quasar nucleus.2 Our $43 \mathrm{GHz}$ observations can resolve the nucleus to within $5 \times 10^{17} \mathrm{~cm}(\sim 0.16 \mathrm{pc})$. It is a fortuitous circumstance that this object is a broad absorption line quasar (BALQSO) as well. About 10\% - 15\% of quasars show broad absorption line (loosely defined as absorbing gas that is blue shifted at least $5,000 \mathrm{~km} / \mathrm{s}$ relative to the QSO rest frame and displaying a spread in velocity of at least 2,000 km/s) outflows (Weymann 1997). It is widely believed that most or all radio quiet quasars have BAL outflows, but the designation of a quasar as a BALQSO depends on whether the line of sight intersects the

\footnotetext{
${ }^{1}$ Throughout this paper, we adopt the following cosmological parameters: $H_{0}=71 \mathrm{~km} / \mathrm{s} / \mathrm{Mpc}, \Omega_{\Lambda}=$ 0.73 and $\Omega_{m}=0.27$ in all our calculations. At $\mathrm{z}=0.042,1$ mas angular separation equates to $0.818 \mathrm{pc}$ displacement in the sky plane in the adopted cosmology.

${ }^{2}$ The radio loudness, $R$, is usually defined as a $5 \mathrm{GHz}$ flux density 10 times larger than the $4400 \AA$ flux density, $R=S_{5 \mathrm{GHz}} / S_{4400 \AA}<10$ (Kellermann et al 1989). This definition is not appropriate, if there is intrinsic extinction as in BALQSOs. One must correct for the intrinsic blue band extinction and recompute the $R$ with dereddened blue fluxes, (Becker et al 2000). Noting the blue band extinction in the text and using the historic $5 \mathrm{GHz}$ flux densities from NED yields a dereddened radio loudness, $1.4<R<3.8$. This is close to the radio intermediate quasar category, $10<R<250$, which are often just Doppler boosted radio quiet quasars (Falcke et al 1996). We show that Doppler boosting greatly enhances $R$ in Mrk 231 as well. The intrinsic radio quiet nature of the AGN in Mrk 231 is not directly apparent because of optical reddening and Doppler boosting.
} 
solid angle subtended by the BAL outflow. Mrk 231 is a LoBALQSO (a BALQSOs that exhibits low ionization resonant absorption troughs). Mrk 231 has broad absorption in a variety of low ionization species, Mg II $\lambda$ 2798, the well known Na I D doublet, He I $\lambda 3889$ I, Ca II K $\lambda 3934$ and Ca II H $\lambda 3968$ (Smith et al 1995; Lipari et al 1994; Martel 1997). Thus, Mrk 231 displays the full panoply of quasar related phenomena, broad emission lines, powerful thermal luminosity, UV broad absorption lines and a radio jet. Therefore, as a consequence of its proximity to earth, Mrk 231 can provide an optimal opportunity to study the interaction of these physical elements by means of high resolution VLBI observations on sub-parsec scales.

Earlier 15 GHz self-calibrated VLBA (Very Long Baseline Array)3 observations reported in Ulvestad et al (1999a,b) with sub mas resolution revealed a double radio source separated by approximately 1 pc. We initiated a program to look for more sub-structure and component motion at higher resolution by means of phase-referenced observations at $22 \mathrm{GHz}$ and $43 \mathrm{GHz}$ VLBA as discussed in section 2. No further resolution into sub-components was achieved even at $43 \mathrm{GHz}$. The two archival epochs showed that the nucleus was very dynamic and set a lower bound on the time variability brightness temperature of $T_{b}>10^{10} \mathrm{~K}$, below the inverse Compton limit, $\sim 10^{12} \mathrm{~K}$ (Ulvestad et al 1999a, b). Thusly motivated, our program of observations attempted short time interval sampling (3 months between the epoch 2006.07 and 2006.32) in order to elucidate this highly dynamic behavior. Mrk 231 did not disappoint, there was an enormous flare between these two epochs. Section 5 is dedicated to analyzing the rapidly changing core. We use our data and the adopted cosmological model to show that the strong flare will violate the constraints associated with the brightness temperature limit unless relativistic Doppler beaming is invoked.

We also explore the steep spectrum secondary component of the nucleus of Mrk 231 in section 6. It is mildly variable and it is just less than a parsec from the core. Based on the nature of the relativistic core, it is natural to wonder if there is any relativistic motion of the secondary. In section 3, we determine that the secondary is either not moving at all or is moving at velocities far below relativistic values. Thus, the more relevant question becomes what is constraining the secondary from having such motion. Section 6 uses our VLBA observations and archival low frequency observations to model the apparent free-free absorption of the secondary emission. We argue that this same gas is confining the secondary by ram pressure and that this gas is likely one and the same as the BAL wind.

\footnotetext{
${ }^{3}$ The National Radio Astronomy Observatory is a facility of the National Science Foundation operated under cooperative agreement by Associated Universities, Inc.
} 


\section{The Observations}

High frequency (15, 22 and $43 \mathrm{GHz}$ ) observations were conducted at epochs 2006.07 (Project ID BP124A) and 2006.32 (Project ID BP124B) with all ten antennas of the VLBA. In addition, data from 2000.02 (Project ID BU013E) at frequencies of 8.4, 15 and $22 \mathrm{GHz}$ and 8.3 GHz data from 2006.67 (Project ID BA080B) were retrieved from the VLBA public archive. The data were correlated on the VLBA correlator in Socorro, NM and calibrated with NRAO's Astronomical Image Processing System (AIPS) software package. In addition to the standard calibration procedure for VLBI polarization observations (Cotton 1993; Leppänen et al. 1995), an attempt was made to correct for amplitude losses due to opacity using the AIPS task APCAL (Leppänen 1993).

At all epochs and frequencies, except 2006.67, the data were initially phase-referenced (Beasley and Conway 1995) to J1302+5748. At frequencies below $43 \mathrm{GHz}$ the data were then phase self-calibrated, but at $43 \mathrm{GHz}$ there was insufficient signal-to-noise to allow this. The phase reference source was imaged to provide the best possible model for the phase referencing procedure, but proved in any case to be a very close approximation to a point source.

Figure 1 captures the images of the $8.4 \mathrm{GHz}$ observations from 2000.02 and 2006.67. Figures 2 and 3 are the images at 15 and $22 \mathrm{GHz}$, respectively, for all three epochs and Figure 4 is the $43 \mathrm{GHz}$ images from 2006.07 and 2006.32. All images of Mrk 231 presented in this paper are made with Briggs' robust weighting scheme (Briggs 1995), with the robustness parameter set to zero, with the exception of the $43 \mathrm{GHz}$ image at epoch 2006.32 where natural weighting is used. All images are centered on the position of the southwestern, secondary, component at rectangular coordinate (0 mas, 0 mas).

Each of the images shows a pair of unresolved components separated by about 1 mas that vary in brightness, but do not vary significantly in separation. The NE component is the most variable and has the flattest spectrum and it is assumed to be associated with the AGN nucleus.

Gaussians were fitted to the positions of the two components using the DIFMAP software package (Shepherd et al. 1994, 1995) and the resultant parameters are presented in Tables 1 and 2, Errors in the flux density in Table 1 arise from two sources: the absolute flux scale error, and an error due to uncertainties in the model fit. The method used to determine the model fit part of the error is from Britzen et al (2007). The absolute flux scale errors for the VLBA are estimated to be $\sim 5 \%$ at $8 \mathrm{GHz}$ (Ulvestad 2008) and $15 \mathrm{GHz}$ (Homan et al 2002), and $\sim 7 \%$ at $22 \mathrm{GHz}$ (Homan et al 2002). At $43 \mathrm{GHz}$ we estimate the error on the absolute flux density scale to $\sim-8 \% /+15 \%$ - the non-symmetric error arising from the fact 
that some flux density is likely lost due to the inability to phase self-calibrate this source at that frequency. An analysis of the amplitude corrections derived from self-calibration of various calibrator sources indicates that these observations were not affected by any unusual amplitude errors that would lead to larger errors than the values quoted above.

Based on the inverted spectrum from epoch 2000.02, we anticipated dramatic behavior on the smallest of scales. Thus, we proposed for two epochs of high frequency observations that were closely spaced in time, 2006.07 and 2006.32. The observations were phase referenced to J1302+5748 at 15, 22 and $43 \mathrm{GHz}$. We also gathered polarization data. There is no evidence that the flux density recovered at any of our frequencies was strongly dependent on the weighting used, indicating that we are dealing with two compact components with little, if any, diffuse flux on the range of spatial scales probed by these observations.

The inability to phase self-calibrate at $43 \mathrm{GHz}$ opens the possibility that some flux density may have been lost due to phase errors. In lieu of the undetected secondary calibrator, J1306+5529, one can use the secondary component to the SW in MRK 231 as a crude amplitude calibrator. Note the flux density of the secondary was the same between epochs 2000.02 and 2006.32 at $22 \mathrm{GHz}$ to within a few percent in Table 1 indicating that it is not strongly variable. Therefore, we treat this component as approximately steady at high frequency and it can potentially serve as a crude calibrator for the core flux density at 43 GHz. The flux density of the secondary (our crude high frequency amplitude calibrator) measured at both epochs at $43 \mathrm{GHz}$ agrees to within $1.2 \mathrm{mJy}$ (0.6 sigma). This supports the suggestion that the phase referencing with respect to $\mathrm{J} 1302+5748$ recovered the majority of the flux at $43 \mathrm{GHz}$, and any amplitude loss due to phase errors is likely to be similar at the two epochs.

We note that in epoch 2006.07 bad weather and technical problems allowed us to recover data from effectively just 5.25 of the 10 array antennas at $43 \mathrm{GHz}$, so these data have sparser $\mathrm{u}-\mathrm{v}$ coverage and an increased RMS noise level. Our $43 \mathrm{GHz}$ images in Figure 4 show partial resolution of the core at epoch 2006.07 with an extension at PA $=135^{\circ}$ from NE to SW. Unfortunately, we must consider this detection unreliable due to the poor beam shape and the low SNR. We stress that the data from all 10 antennas were recovered at $43 \mathrm{GHz}$ at epoch 2006.32. This higher quality data is used extensively in section 5 .

In Figures 5 and 6, we plot the spectrum of the core and the secondary, respectively, for all three epochs of observation as well as the two archival data sets in Ulvestad et al $(1999 \mathrm{a}, \mathrm{b})$.

The core spectra in Figure 5 are highly variable in magnitude. All the epochs reveal an optically thick core that is indicated by flat or inverted spectra in a finite band of frequency. 

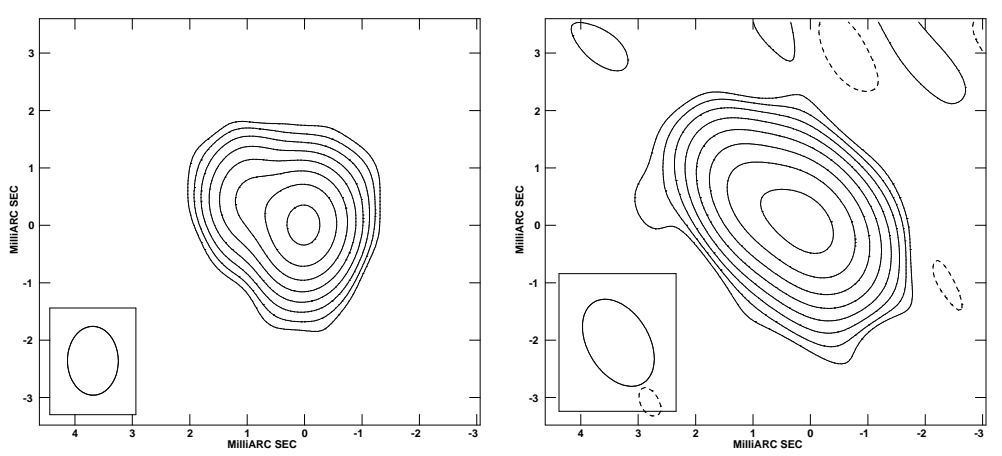

Fig. 1. - VLBA $8 \mathrm{GHz}$ images of Mrk 231 from epoch 2000.02 (left) and epoch 2006.67 (right). The contours start at $0.7 \mathrm{mJy} /$ beam and increase in factors of 2 to a maximum of $89.6 \mathrm{mJy} /$ beam.
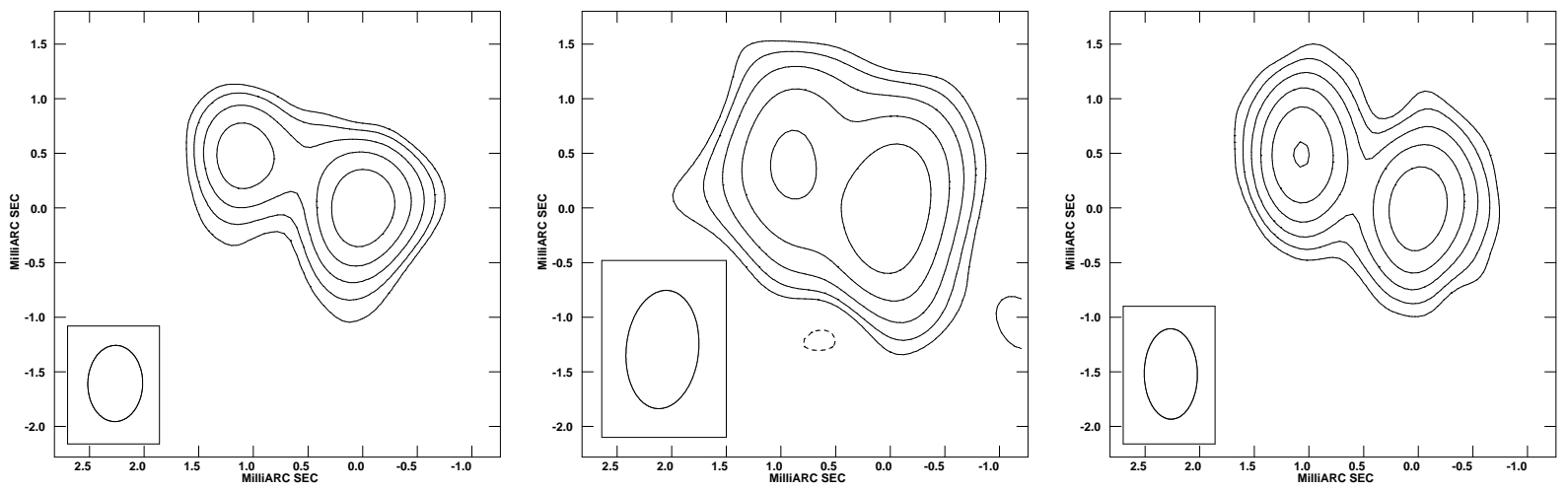

Fig. 2.- VLBA $15 \mathrm{GHz}$ images of Mrk 231 from epoch 2000.02 (left), epoch 2006.07 (middle) and epoch 2006.32 (right). The contours start at $1.8 \mathrm{mJy} /$ beam and increase in factors of 2 to a maximum of $57.6 \mathrm{mJy} /$ beam.
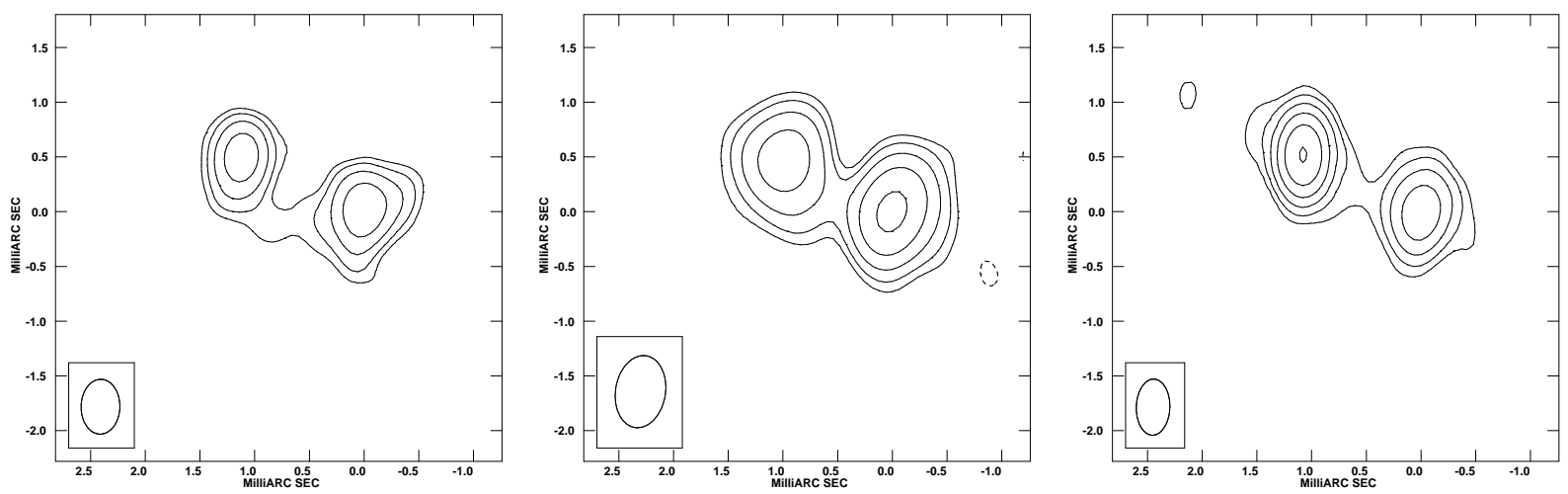

Fig. 3.- VLBA 22 GHz images of Mrk 231 from epoch 2000.02 (left), epoch 2006.07 (middle) and epoch 2006.32 (right). The contours start at $1.8 \mathrm{mJy} /$ beam and increase in factors of 2 to a maximum of $57.6 \mathrm{mJy} /$ beam. 

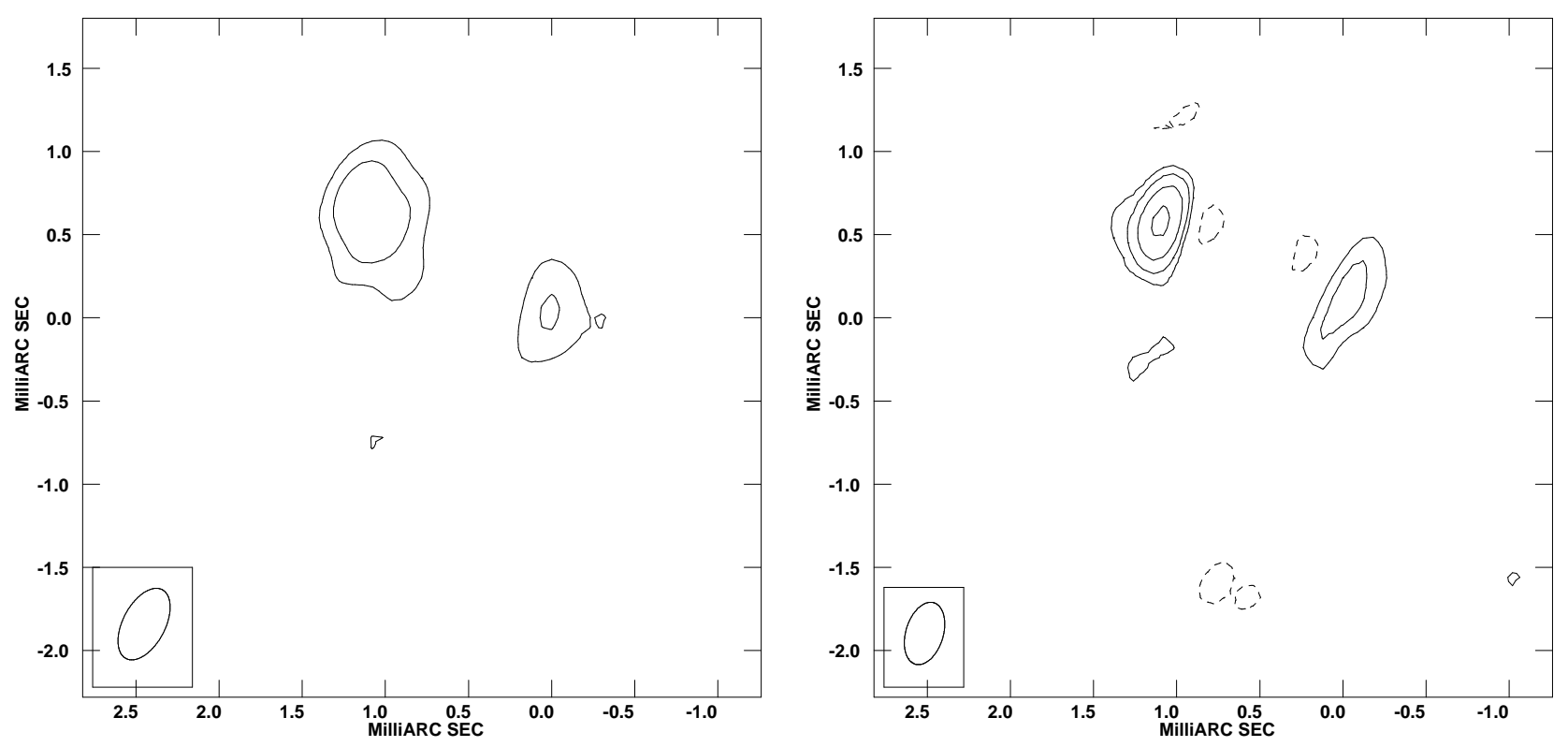

Fig. 4.- VLBA $43 \mathrm{GHz}$ images of Mrk231 from epoch 2006.07 (left) and epoch 2006.32 (right). The contours start at $2.2 \mathrm{mJy} /$ beam and increase in factors of 2 to a maximum of $17.6 \mathrm{mJy} /$ beam.

Table 1: High Frequency VLBA Observations Data Summary

\begin{tabular}{cccccc}
\hline Component & Date & $\begin{array}{c}8.4 \mathrm{GHz} F l u x \\
\text { Density }(\mathrm{mJy}) / \% \mathrm{P}^{\mathrm{a}}\end{array}$ & $\begin{array}{c}15 \mathrm{GHz} \text { Flux } \\
\text { Density }(\mathrm{mJy}) / \% \mathrm{P}^{\mathrm{a}}\end{array}$ & $\begin{array}{c}22 \mathrm{GHz} \text { Flux } \\
\text { Density }(\mathrm{mJy}) / \% \mathrm{P}^{\mathrm{a}}\end{array}$ & $\begin{array}{c}43 \mathrm{GHz} \text { Flux } \\
\text { Density }(\mathrm{mJy}) / \% \mathrm{P}^{\mathrm{a}}\end{array}$ \\
\hline $\mathrm{NE}$ & 1996.93 & $18 \pm 2$ & $17 \pm 2$ & $\ldots$ & $\ldots$ \\
$\mathrm{NE}$ & 1998.71 & $\ldots$ & $44 \pm 3$ & $\ldots$ & $\ldots$ \\
$\mathrm{NE}$ & 2000.02 & $29.1 \pm 1.5 /<1.0$ & $31.3 \pm 1.8 /<1.9$ & $26.7 \pm 2.0<3.9$ & $\ldots$ \\
$\mathrm{NE}$ & 2006.07 & $\ldots$ & $43.1 \pm 2.2 /<1.9$ & $29.4 \pm 2.2 /<3.4$ & $20.1_{-1.9}^{+3.2} /<13.7$ \\
$\mathrm{NE}$ & 2006.32 & $\ldots$ & $68.6 \pm 3.5 /<1.2$ & $74.8 \pm 5.3 /<0.7$ & $21.3_{-1.8}^{+3.3} /<10.1$ \\
$\mathrm{NE}$ & 2006.67 & $57.0 \pm 3.9 / \ldots$ & $\ldots$ & $\ldots$ & $\ldots$ \\
\hline $\mathrm{SW}$ & 1996.93 & $114 \pm 11$ & $51 \pm 3$ & $\ldots$ & $\ldots$ \\
$\mathrm{SW}$ & 1998.71 & $\ldots$ & $60 \pm 3$ & $\ldots$ & $\ldots$ \\
$\mathrm{SW}$ & 2000.02 & $126.2 \pm 6.3 /<0.24$ & $71.2 \pm 3.7<0.28$ & $36.5 \pm 2.7<2.9$ & $\ldots$ \\
$\mathrm{SW}$ & 2006.07 & $\ldots$ & $69.5 \pm 3.5 /<1.2$ & $43.1 \pm 3.1 /<1.3$ & $9.8_{-1.3}^{+1.8} /<28.1$ \\
$\mathrm{SW}$ & 2006.32 & $\ldots$ & $66.8 \pm 3.4 /<1.3$ & $40.0 \pm 2.9 /<9$ & $8.6_{-0.9}^{+1.4} /<25.1$ \\
$\mathrm{SW}$ & 2006.67 & $127.5 \pm 6.3 / \ldots$ & $\ldots$ & $\ldots$ & $\ldots$ \\
\hline
\end{tabular}

${ }^{a}$ The polarization is a $3 \sigma$ upper limit 
Table 2: Component Separation

\begin{tabular}{|c|c|c|c|c|c|c|c|c|c|c|c|c|}
\hline Date & $8.4 \mathrm{GHz}^{\mathrm{a}}$ & $\sigma_{1}{ }^{\mathrm{b}}$ & $\sigma_{2}{ }^{\mathrm{c}}$ & $15 \mathrm{GHz}^{\mathrm{a}}$ & $\sigma_{1}{ }^{\mathrm{b}}$ & $\sigma_{2}{ }^{\mathrm{c}}$ & $22 \mathrm{GHz}^{\mathrm{a}}$ & $\sigma_{1}{ }^{\mathrm{b}}$ & $\sigma_{2}{ }^{\mathrm{c}}$ & $43 \mathrm{GHz}^{\mathrm{a}}$ & $\sigma_{1}{ }^{\mathrm{b}}$ & $\sigma_{2}{ }^{\mathrm{c}}$ \\
\hline 1996.93 & $\ldots$ & $\ldots$ & $\ldots$ & 1.081 & 0.03 & 0.172 & $\ldots$ & $\ldots$ & $\ldots$ & $\ldots$ & $\ldots$ & $\ldots$ \\
\hline 1998.71 & & & & 1.162 & 0.004 & 0.174 & & & & $\ldots$ & $\cdots$ & $\ldots$ \\
\hline 2000.02 & 1.038 & 0.004 & 0.126 & 1.173 & 0.003 & 0.139 & 1.219 & 0.005 & 0.128 & & & \\
\hline 2006.07 & $\ldots$ & $\ldots$ & $\ldots$ & 1.009 & 0.006 & 0.172 & 1.093 & 0.005 & 0.132 & 1.222 & 0.013 & 0.142 \\
\hline 2006.32 & $\ldots$ & 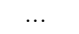 & $\ldots$ & 1.172 & 0.002 & 0.089 & 1.193 & 0.002 & 0.059 & 1.216 & 0.010 & 0.103 \\
\hline
\end{tabular}

${ }^{a}$ Measured separation at the designated frequency in mas

${ }^{b} \sigma_{1}$ is the error computed using the technique of Condon et al (1998), see equation (1)

${ }^{c} \sigma_{2}$ is the error computed using the technique of Lobanov (2005), see equation (2)

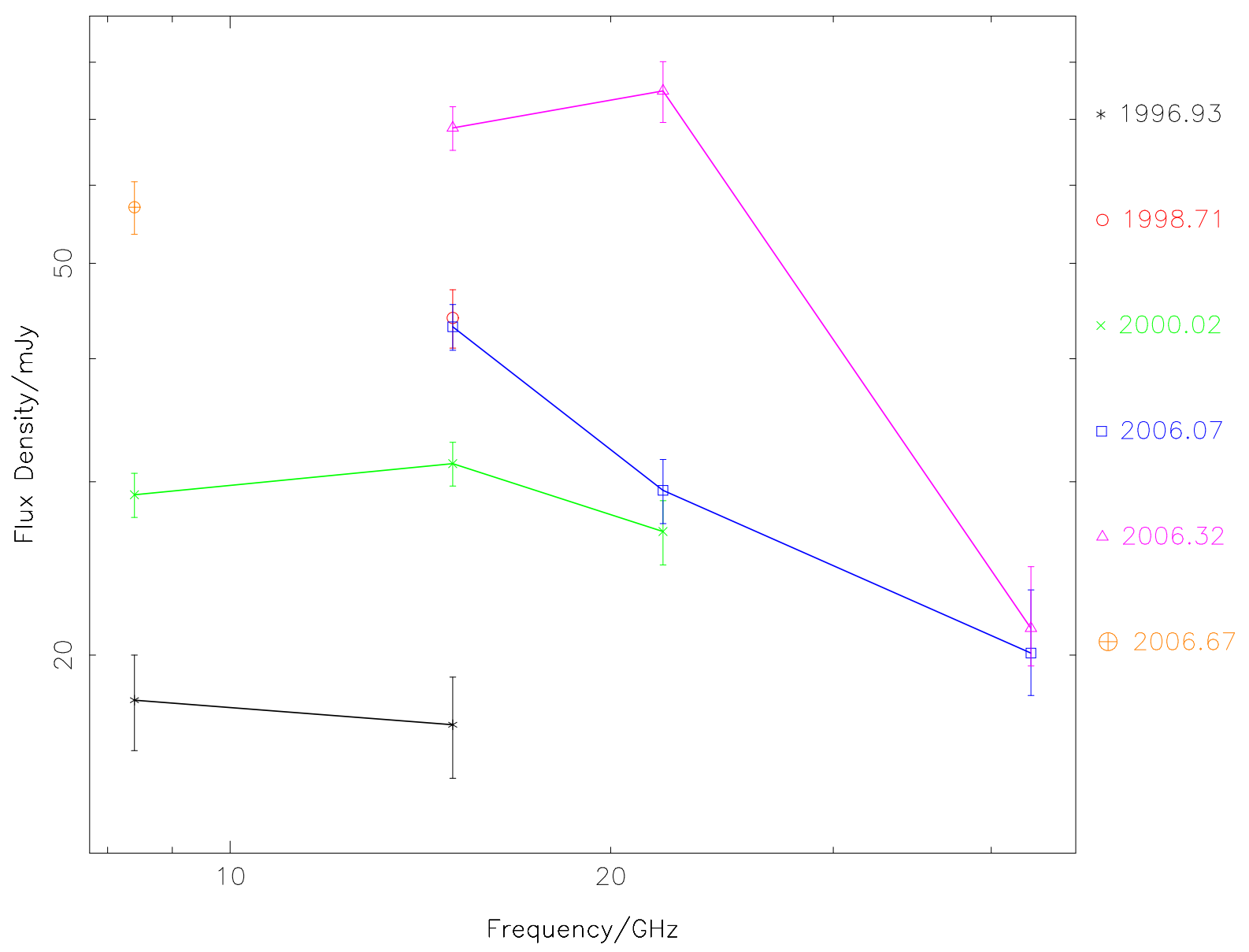

Fig. 5.- The core spectra of Mrk 231 at six different epochs of VLBA observation. Notice that the data point for 1998.71 overlaps the $15 \mathrm{GHz}$ point at epoch 2006.07. 
Clearly the most powerful epoch is 2006.32 which we explore in detail in section 5 . In contrast Figure 6 shows that the secondary is optically thin, steep spectrum at all epochs. The data are supportive of a secondary that is mildly variable, characterized by changes of $<10 \%$ $(<1 \sigma)$ on time scales of 0.25 years, $\sim 15 \%(<2 \sigma)$ on time scales of 6 years and $\sim 30 \%$ on time scales of 9.4 years. Notice that the spectra show significant curvature, reflecting a deficit of high frequency flux relative to a power law extrapolated from lower frequency.

\section{Component Motions and Positions}

An important part of this analysis is to try to determine the motion of the secondary relative to the core over the 9.4 year history of VLBA observations that were capable of resolving the compact nuclear double (i.e., observations at $\geq 15 \mathrm{GHz}$ ). The entries in Tables 1 and 2 include the historical data from Ulvestad et al $(1999 \mathrm{a}, \mathrm{b})$ for this reason. It is clear that there is very little component motion. Therefore, a careful analysis of the errors that arise in the determination of the component separation is critical. The displacements between the core and secondary are entered in Table 2. Those entries in the first five rows have errors computed by a method that is based on the error in discerning the position of the centroid of an elliptical Gaussian fit to the data in the presence of background noise (e.g., Condon et al (1998)),

$$
\Delta \theta_{\text {sep }}=0.5\left[\left(\frac{\theta_{\text {beam }}}{S N R_{\text {core }}}\right)^{2}+\left(\frac{\theta_{\text {beam }}}{S N R_{\text {secondary }}}\right)^{2}\right]^{0.5}
$$

where $\Delta \theta_{\text {sep }}$ is the error in the estimate of the separation in mas, $\theta_{\text {beam }}$ is the beamwidth in mas and SNR is the signal to noise ratio. Notice that the component separations in Table 2 widen with increasing observing frequency at each epoch as expected for an optically thick core (Lobanov 1998; Konigl 1981). Thus, in computing a change in the component separation between epochs a common frequency should be used. The lone common frequency of observation for all epochs is $15 \mathrm{GHz}$.

However, the method described by equation (1) does not account for core subcomponents that flare on scales below the resolution limit of the beam without any actual motion occurring. If a subcomponent flares, the centroid of the elliptical fit of the core can shift in position and give the illusion of secondary motion. This is a concern for Mrk 231 since Figure clearly indicates the presence of strong core flares that are not resolved by VLBI. For example, the core flux density flares at 1998.71 relative to the flux density of the core at 1996.93 and the components appear to separate by 0.08 mas at $15 \mathrm{GHz}$ according to Table 2. This can be described by the base of the jet at the far eastern edge of the core being the 


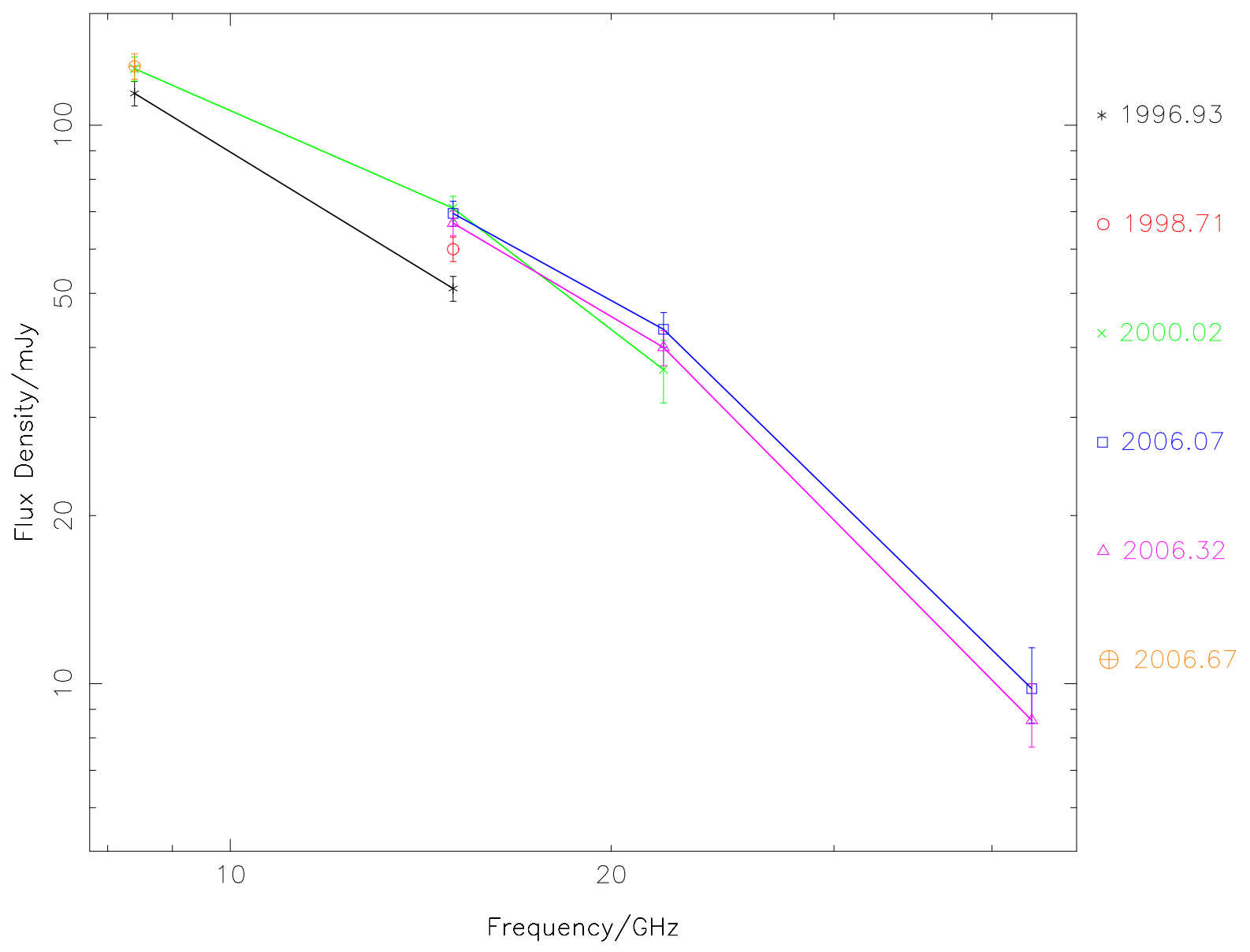

Fig. 6. - The spectra of the secondary component of the nucleus of Mrk 231 at six different epochs of VLBA observation. 
source of the flare with no actual secondary motion. The position of the elliptical fit just shifts to the east by about 0.08 mas, well below the resolution of the beam at $15 \mathrm{GHz}$ or $22 \mathrm{GHz}$ where the flare is detected. Thus, we need an estimate of the error in component separation that incorporates the maximum size subcomponent flare in the core that can not be resolved with VLBI (equivalently, the minimum size subcomponent that can be resolved by VLBI). Comparing the maps of the compact double in Ulvestad et al (1999a) at 15.3 $\mathrm{GHz}$ (resolved), $8.4 \mathrm{GHz}$ (partially resolved) and $5 \mathrm{GHz}$ (unresolved), it appears that the uncertainty posed by unresolved subcomponents in the core will be about $1 / 2-1 / 3$ of the beamwidth projected along the direction toward the secondary. Fortuitously for the compact double in Mrk 231 this direction is close to the minor axis of the beam. In general, the minimum size subcomponent that is resolvable by VLBI will depend on the SNR, so we look for a more general and quantitative formulation. To this end we invoke the technique of Lobanov (2005) that considers the ability of an interferometer to resolve components modeled by elliptical fits in presence of noise assuming a rectangular power law interferometric visibility sampling function,

$$
\theta_{\lim }=\frac{2^{2-\beta / 2}}{\pi}\left[\pi a b \ln 2 \ln \left(\frac{S N R}{S N R-1}\right)\right]^{0.5} .
$$

In equation (2), $\theta_{\lim }$ is the minimum size subcomponent in mas that can be resolved by the interferometer. In our calculations we have the weighting of the data, $\beta=0$ corresponding to unweighted data. The major axis and minor axis of the beam in mas are $a$ and $b$, respectively. The values of $\theta_{\text {lim }}$ that we derive from equation (2) range from $b / 4-b / 2$ for the observations in Table 2. We consider, $\theta_{\lim }$ an estimate of the uncertainty in the core position due to unresolved flaring subcomponents. The secondary does not flare, so we do not consider this a likely source of error in the position of the secondary. We add this error to the error introduced in equation (1) in quadrature $\left(\theta_{\lim }\right.$ is the dominant contributor). The results computed by the formula, (2), are listed for the separation error on rows 6 - 10 of Table 2 . They are large compared to the variations in the component separation between any pair of epochs. We conclude that the observations in Table 2 are not precise enough to determine the rate of component separation, except as an upper limit determined by $\theta_{\text {lim. }}$ The VLBA observations indicate that the separation in components changed in 9.4 years (from 1996.93 to 2006.32 ) by

$$
\Delta \theta=0.091 \pm 0.094 \text { mas }
$$

In arriving at the result given by equation (3), we noted that the core at epoch 1996.93 was at an extreme historic low. Thus, we do not consider core flares relevant in this quiescent state. Therefore, we use the error derived from equation (1) that appears in the first row of Table 2 as an accurate assessment of the error in the measured component separation at this 
epoch. At 2006.32, there is clearly a flare, so we use the $15 \mathrm{GHz}$ entry derived from equation (2) that appears in row 10 of Table 2 to estimate the error in the component separation at this epoch. The secondary separation in equation (3) equates to a time averaged secondary separation velocity, $v_{a d v}$

$$
v_{a d v}=0.026 c \pm 0.027 c .
$$

Our data is consistent with no apparent motion of the secondary relative to the core. It should be remembered that $v_{a d v}$ is the projection of the separation velocity of the components onto the sky plane.

\section{The Core Structure at 2006.07}

Table 2 indicates that the component separation increases with observing frequency at all epochs, This is expected in optically thick cores as the $\tau=1$ surface for synchrotron self absorption moves outward relative to the true core position with decreasing frequency (Konigl 1981). In order to ascribe a quantitative measure of this phenomena one must resort to highly model dependent estimates that involve much speculation about many unknown physical quantities that parameterize the putative jet (Lobanov 1998). However, notice that this variation is particularly pronounced at 2006.07. Furthermore, from Figure 5, the core is actually optically thin between $15 \mathrm{GHz}$ and $22 \mathrm{GHz}$, yet there is an $84 \mu$ as differential in the component separations. In this section we study the ramifications of this to see if we can make some quantitative statements with a minimal amount of model dependent assumptions.

We begin by overlaying the component positions on the $43 \mathrm{GHz}$ radio image at epoch 2006.07 in Figure 7. The main issue is how does one register the image centers at different observing frequencies. This was not designed as an astrometric experiment and we do not have a good handle on the positional jitter that is induced by fluctuations in the tropospheric delay.

Ostensibly there is no obvious way to place errors on the component positions in Figure 7. However, notice that the secondary components are fairly close in position. In fact we can argue that they should be even closer than this based on the steep spectrum in Figure 6. We seek to register all of the epoch 2006.07 radio images relative to the $22 \mathrm{GHz}$ position of the unresolved secondary at 2006.07. Let $\Sigma_{22}$ be the region containing the plasma that emits the bulk of the $22 \mathrm{GHz}$ secondary emission. For any reasonable ensemble of energetic particles, there must also be emission at $43 \mathrm{GHz}$ and $15 \mathrm{GHz}$. First, consider the $43 \mathrm{GHz}$ emission. The spectral index in Figure 6 between $22 \mathrm{GHz}$ and $43 \mathrm{GHz}$ is incredibly steep, $\alpha_{43}^{22}=2.2$, where the spectral index is defined in terms of the flux density by the convention, 

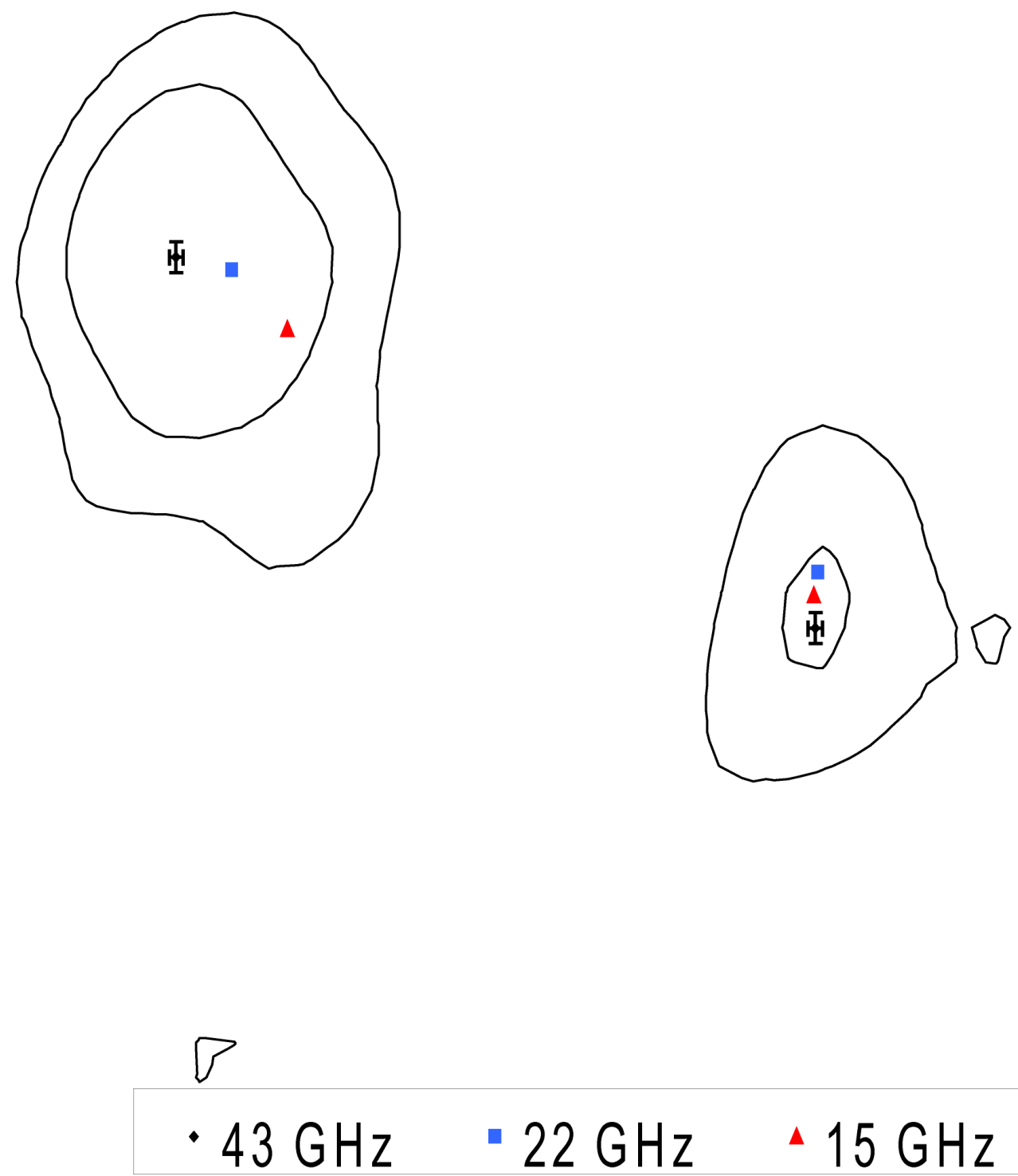

Fig. 7.- An overlay of the frequency dependent component positions on the $43 \mathrm{GHz}$ image at epoch 2006.07. The errors in positions are naively computed using equation (1). Only the $43 \mathrm{GHz}$ points have significant error bars. A more accurate error assessment method is shown in Figure 8 and discussed in the text. 
$S_{\nu} \sim \nu^{-\alpha}$. Between $15 \mathrm{GHz}$ and $22 \mathrm{GHz}$ the spectral index is significantly flatter, but still very steep, $\alpha_{22}^{15}=1.2$. Thus, unless there is an incredibly abrupt spectral break above 22 $\mathrm{GHz}$ and a second weak $43 \mathrm{GHz}$ component, virtually all of the $43 \mathrm{GHz}$ radiation from the secondary would be emitted from the particles in $\Sigma_{22}$. Therefore, we consider the assumption that the $43 \mathrm{GHz}$ emitting electrons are co-spatial with the $22 \mathrm{GHz}$ emitting plasma is most likely valid. We can not make the same argument for the $15 \mathrm{GHz}$ emitting electrons. Since the spectrum is so steep between 43 and $22 \mathrm{GHz}$ and the spectrum above $22 \mathrm{GHz}$ is also steep, we make the conservative assumption that the $15 \mathrm{GHz}$ flux density from $\Sigma_{22}$ is at least as big as the $22 \mathrm{GHz}$ flux density, $43.1 \mathrm{mJy}$. With this conservative assessment, we can estimate an upper bound on the error in the $15 \mathrm{GHz}$ secondary position if it is re-registered co-spatially with the $22 \mathrm{GHz}$ secondary position. Namely, if the remaining $26.4 \mathrm{mJy}$ of residual $15 \mathrm{GHz}$ flux resides primarily in a disjoint component, $\Sigma_{15}$, this would create the maximum offset in the true absolute position of the $15 \mathrm{GHz}$ component position relative to $\Sigma_{22}$. The $15 \mathrm{GHz}$ secondary is unresolved in Figure 2, and according to Ulvestad et al (2005) and our own experience discussed in section 3, if $\Sigma_{15}$ was half a beam width away from $\Sigma_{22}$, the secondary would be partially resolved in the radio map at $15 \mathrm{GHz}$. There is no partially resolved component in Figure 2. Thus, we can set an upper bound on the error in registering the $15 \mathrm{GHz}$ secondary position to the $22 \mathrm{GHz}$ position, by positing that 26.4 mJy of flux density arise from an optically thin component, $\Sigma_{15}$, that lies one half of a beam width from $\Sigma_{22}$ (which accounts for the remaining $43.1 \mathrm{mJy}$ of the $15 \mathrm{GHz}$ flux density of the secondary). Then taking the flux density weighted average of the component positions (i.e., offset from $\left.\Sigma_{22}=(26.4 / 69.5)(\theta / 2)\right)$ yields a conservative upper bound on the offset between the true $15 \mathrm{GHz}$ position and the $22 \mathrm{GHz}$ position. The resultant upper bound on the error in the re-registered $15 \mathrm{GHz}$ position is 0.11 mas and 0.018 mas in the $\mathrm{x}$ and $\mathrm{y}$ directions, respectively. Figure 8 is the Figure 7 overlay re-registered to the $22 \mathrm{GHz}$ secondary position (i.e., all three of the secondary positions agree). The errors on the re-registered 22 and 43 $\mathrm{GHz}$ core data points in Figure 8 arise from equation (1). The error on the $22 \mathrm{GHz}$ core position is too small to be seen in this figure.

The re-registered map in Figure 8 makes the component positions appear almost linear. We believe that our assignment of errors justifies further analysis of the frequency dependent component separation data. Apparently, we have uncovered sub-structure on scales smaller than the $43 \mathrm{GHz}$ beamwidth. But, unlike the optically thick case in which the core position movement reflects the location of the frequency dependent $\tau=1$ surface, the 2006.07 core spectrum is optically thin below $22 \mathrm{GHz}$ and we are revealing direct data on the distribution of the synchrotron emitting gas. In particular, the spread in the $15 \mathrm{GHz}$ and $22 \mathrm{GHz}$ core positions in Figure 8, reflects the fact that there are significant amounts of $22 \mathrm{GHz}$ emitting plasma to the east of the plasma that emits the $15 \mathrm{GHz}$ data. In fact, the spectrum in Figure 


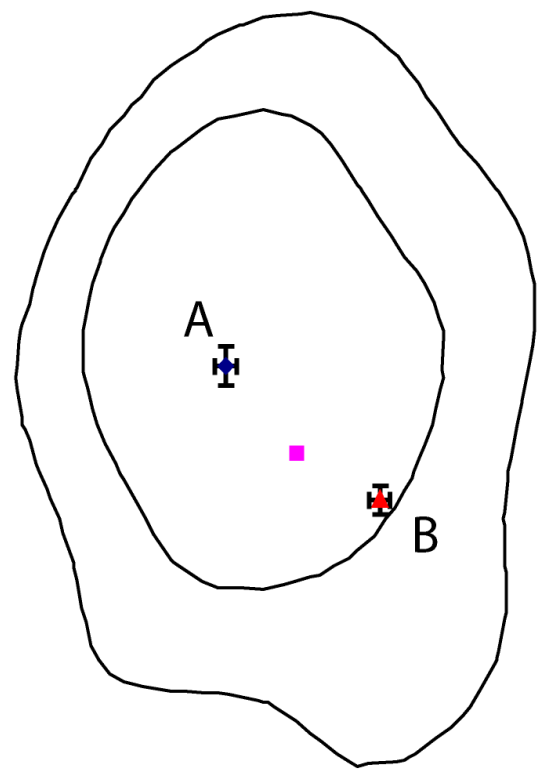

\section{- $43 \mathrm{GHz}=22 \mathrm{GHz} \triangle 15 \mathrm{GHz}$}

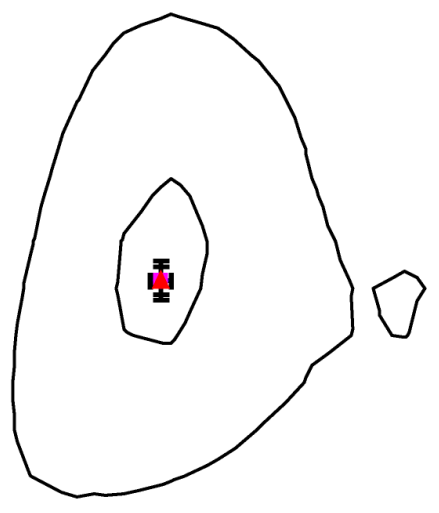

Fig. 8. - An overlay of the frequency dependent component positions on the $43 \mathrm{GHz}$ image at epoch 2006.07. This figure is similar to Figure 7, except that the components are reregistered relative to the $22 \mathrm{GHz}$ secondary position. This tells us how the map center at one frequency is related to the map centers at the other frequencies. To get an absolute registration within the $43 \mathrm{GHz}$ radio image, we place the 3 coincident secondary positions at the location of the best fit Gaussian to the $43 \mathrm{GHz}$ secondary flux density. The A and B are labels that show the component locations within the theoretical two component model. Component A is synchrotron self absorbed and component B is very steep spectrum. 
5 and core component positions are well represented by a two component model. Component A is located $\approx 40 \mu$ as to the northeast of the $43 \mathrm{GHz}$ core position, along a line connecting the three core position components and component $\mathrm{B}$ is $\approx 40 \mu$ as to the southwest of the 15 $\mathrm{GHz}$ core position along the same axis. Component $\mathrm{A}$ is synchrotron self absorbed with a peak flux density of $\approx 27 \mathrm{mJy}$ at $\approx 32 \mathrm{GHz}$. Component B is steep spectrum with $\alpha \approx 2.4$. We only introduce the model to give a qualitative feel for the underlying substructure not to fine tune the parameters. This model explains the inflection point in the SED at epoch 2006.07 inferred from Figure 5 and predicts $\approx 3 \mathrm{mJy}$ at $43 \mathrm{GHz}$ in component $\mathrm{B}$, similar to what we see in the southwest core extension in the radio image in Figure 4. Again, we caution that this feature might just be phase noise. Without a higher signal to noise detection, we can not say more. However, the spread in component positions and the SED are consistent with there being some emission in this region.

The reason for pursuing this two component model in Figure 8 is not to make precise statements about the components that are not directly detected, but to explore the more general implications of a core substructure at 2006.07, composed of an optically thick component and a steep spectrum component, that is required to fit the SED. To this end, we plot the SED of the theoretical components A and B relative to the SEDs at 2006.07 and 2006.32 in Figure 9.

The first deduction is that the flare at 2006.32 can not be created by the simple adiabatic expansion of the flaring core component $\mathrm{A}$ at 2006.07. If it were simple adiabatic expansion of a flare that is peaked at $32 \mathrm{GHz}$ at 2006.07, then the optically thin flux at $43 \mathrm{GHz}$ should be much weaker at 2006.32, (Moffet 1975), but it is actually stronger. Thus, the flare at 2006.32 requires a large injection of energetic particles and energy after 2006.07.

The second important implication is that component B is very steep spectrum above 15 GHz. Figure 9 shows that there is some mechanism that causes the high energy spectrum to decay rapidly in the core of Mrk 231. All the components are extremely steep in the optically thin region, thus there is a very efficient cooling mechanism that preferentially cools the particles with the highest energy first. The most common mechanism in AGN that fit this prescription are inverse Compton cooling from a strong radiation field or synchrotron emission from a strong magnetic field. The cooling is so efficient that by the time that an ejection from the nucleus is far enough away so that it could be resolved by $43 \mathrm{GHz}$ VLBA, it is generally too weak to be detected. Even with all 10 antennas working at peak efficiency, two good calibrators at $43 \mathrm{GHz}$ and good weather, partial resolution of the ejecta might be the best one can do when studying flare evolution in the nucleus of Mrk 231.

The next section is the main topic of this study. We explore the formation of the powerful flare at 2006.32. Furthermore, we try to get an understanding of the rapid cooling 


\section{THE CORE SED in the TWO COMPONENT MODEL}

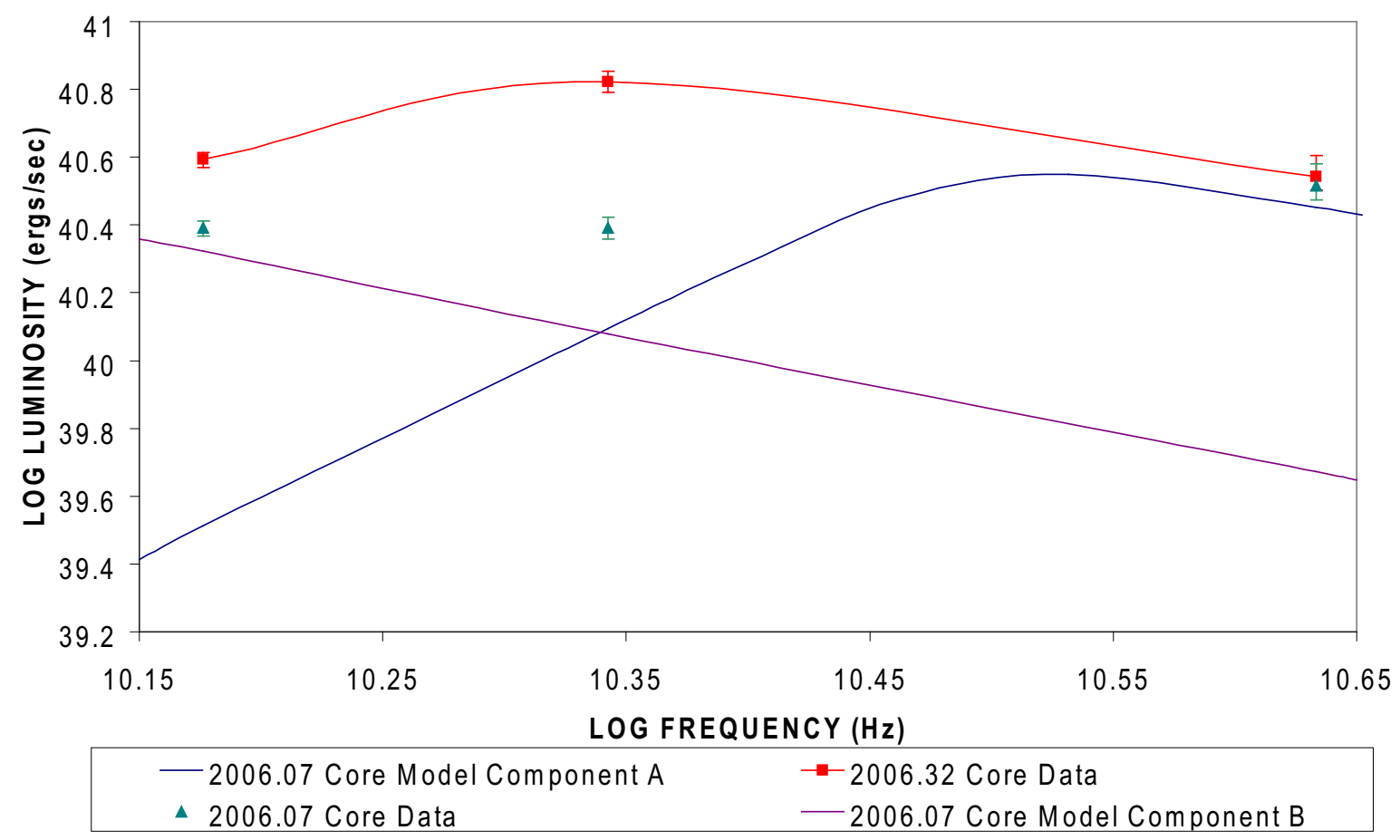

Fig. 9. - Comparison of SEDs of the theoretical two component model of the core at 2006.07 with the SED at 2006.32 
mechanism that causes drastic temporal changes in the emissivity of the ejected flares.

\section{The Core Flare at Epoch 2006.32}

The data in Table 1 indicates that the NE component is highly variable and is likely associated with the AGN nucleus. The two epochs of the observations, 2006.07 and 2006.32, reveal a strong flare at $22 \mathrm{GHz}$ that increased the flux density by $45 \mathrm{mJy}$ in just 91 days. The inverted spectrum from $15 \mathrm{GHz}$ to $22 \mathrm{GHz}$ juxtaposed to the steep spectrum, $\alpha=1.8$, from $22 \mathrm{GHz}$ to $43 \mathrm{GHz}$ is extremely unusual for a radio source and provides a strong constraint on the nature of the flare that we exploit in this section. The steep spectrum above $22 \mathrm{GHz}$ is inconsistent with free-free emission unless the temperature of the gas is $<10^{\circ} \mathrm{K}$ which is unlikely within a few pc of a quasar nucleus. Thus, we consider synchrotron radiation as a more likely source of the emission.

There are two possible explanations for the inverted spectrum near $22 \mathrm{GHz}$. First, the spectral luminosity, $L_{\nu}$, might be seen through a free-free absorbing (FFA) screen. For a powerlaw synchrotron spectrum seen through a FFA gas with number density of free electrons, $N_{e}$, temperature, $T$, and thickness in parsecs, $L_{p c}$, we adopt the following expressions from Walker et al (2000),

$$
\begin{aligned}
& L_{\nu}=L_{0} \times\left(\frac{\nu}{2.2 \times 10^{10} \mathrm{~Hz}}\right)^{\alpha}\left[(1-f)+f e^{-\kappa(\nu)}\right] \\
& \kappa(\nu)=9.8 \times 10^{-3} L_{\mathrm{pc}} N_{e}^{2} T^{-1.5} \nu^{-2}\left[17.7+\ln \left(T^{1.5} \nu^{-1}\right)\right] .
\end{aligned}
$$

We have only three data points in Table 1, the flux densities at 15,22 and $43 \mathrm{GHz}$ of the core. If we rewrite the free-free opacity as $\kappa(F F A) \equiv \kappa_{o} \nu^{-2}\left[17.7+\ln \left(T^{1.5} \nu^{-1}\right)\right]$, we can separate out the frequency dependence, where the coefficient $\kappa_{o} \equiv 9.8 \times 10^{-3} L_{\mathrm{pc}} N_{e}^{2} T^{-1.5}$. We assume that the emission region is a homogeneous sphere ejected along the jet direction toward the secondary. This is certainly more accurate than a continuous jet for emission that shows no evidence in Figure 5 or Table 1 of being quasi-steady. The lack of protracted periods of quasi-steady flux is in contradiction to what would be expected from a large smoothly varying structure, like the jet models in Konigl (1981). Every indication is that the emission around 2006.32 is in the form of a large impulsive burst on the background of some low luminosity feature (perhaps the continuous jet). The episodic flaring nature of the core indicated in Section 4, Figure 5 and Table 1 is consistent with large ejections of highly energized plasma, with the epoch 2006.32 being the most energetic. The epoch 2006.32 plasmoid is likely oblong and irregular with an inhomogeneous density, but without any prior knowledge of these geometric properties, we use a spherical solid as a zeroth order approximation. With this assumption of a homogenous sphere, the coefficient $\kappa_{o}$ is a constant 
in our calculations. We also assume for now that the covering factor, $f=1$. For every value of $T$, we have three variables in equations (5) and (6), $\kappa_{o}, \alpha$ and the normalization $L_{o}$. Thus, we can simultaneously solve for the three variables as three equations (the 3 measured flux densities converted into spectral luminosities in equation (5)) with 3 unknowns (the 3 variables) and the resultant will fit the three flux density data points perfectly. We find the unrealistic result that $\alpha \approx 3.1$ for the broad range of temperatures $10^{3} \mathrm{~K}<T<5 \times 10^{5} \mathrm{~K}$. The extremely steep background spectrum is a consequence of the fact that the observed spectrum is very steep just beyond the spectral break, $\alpha \approx 1.8$ from $22 \mathrm{GHz}$ to $43 \mathrm{GHz}$. If it is the FFA absorbing gas that creates the spectral peak between $15 \mathrm{GHz}$ and $22 \mathrm{GHz}$ in Table 1 then it necessarily has a strong effect on the spectral slope at $22 \mathrm{GHz}$. In particular, since $\kappa(F F A)$ is a function of $\nu$, the attenuation is highly significant at $22 \mathrm{GHz}$. Consequently, the background synchrotron spectrum at $\approx 22 \mathrm{GHz}$ would have to be appreciably steeper than indicated by the observed value of $\alpha=1.8$.

Secondly, the spectral luminosity might be a consequence of radiative transfer through a synchrotron self absorbed (SSA) plasma. The strategy is to perform the final calculation in the plasma rest frame using known variables from observation. We designate the observed quantities with a subscript, "o", in the following expressions. Taking the standard result for the SSA attenuation coefficient in the plasma rest frame and noting that $\nu=\nu_{o} / \delta$, we find from Revnolds et al (1996); Ginzburg and Syrovatskii (1969),

$$
\begin{aligned}
& \mu(\nu)=\frac{3^{\alpha+1} \pi^{0.5} g(p) e^{2} N_{\Gamma}}{8 m_{e} c}\left(\frac{e B}{m_{e} c}\right)^{(1.5+\alpha)} \nu_{o}^{(-2.5+\alpha)} \delta^{(2.5+\alpha)}, \\
& g(n)=\frac{\Gamma[(3 n+22) / 12] \Gamma[(3 n+2) / 12] \Gamma[(n+6) / 4]}{\Gamma[(n+8) / 4]}
\end{aligned}
$$

The Doppler factor, $\delta$, is given in terms of $\Gamma$, the Lorentz factor of the outflow; $\beta$, the three velocity of the outflow and the angle of propagation to the line of sight, $\theta ; \delta=1 /[\Gamma(1-$ $\beta \cos \theta)$ ] (Lind and Blandford 1985). This equation derives from an assumed powerlaw energy distribution for the relativistic electrons, $N(\gamma)=N_{\Gamma} \gamma^{-n}$, where $\gamma$ is the thermal Lorentz factor and the radio spectral index $\alpha=(n-1) / 2$. The radiative transfer equation was trivially solved in Ginzburg and Syrovatskii (1969) to yield the following parametric form for $L_{\nu}$ from the SSA source,

$$
L_{\nu}=\frac{L_{0} \nu^{-\alpha}}{\mu(\nu)} \times\left(1-e^{-\mu(\nu) R}\right)
$$

where $\mathrm{R}$ is the radius of the spherical region in the rest frame of the plasma. We can make the same decomposition that we made for $\kappa(F F A)$ for the SSA attenuation coefficient, $\mu(S S A)=\mu_{o} \nu_{o}^{(-2.5+\alpha)}$. If we assume that the source is spherical and homogeneous then we have three variables in $(9), \mu_{o}, \alpha$ and $L_{o}$. Since we have three equations (the 3 measured 
flux densities converted into spectral luminosities in equation (9)) with 3 unknowns (the 3 variables), we can fit the three flux density measurements perfectly. We find that $\alpha=2.26$ (or $n=5.52$ ). Although this is incredibly steep for a radio source it is still much more reasonable than what we found for FFA. Thus, we consider SSA a much likelier interpretation of the peak in the synchrotron spectrum than FFA.

Thusly motivated, we proceed to detail the spherical, homogeneous SSA model of the core. The synchrotron emissivity is given in Tucker (1975) as

$$
\begin{aligned}
& j_{\nu}=1.7 \times 10^{-21}\left(4 \pi N_{\Gamma}\right) a(n) B^{(1+\alpha)}\left(4 \times 10^{6} / \nu\right)^{\alpha} \\
& a(n)=\frac{\left(2^{\frac{n-1}{2}} \sqrt{3}\right) \Gamma\left(\frac{3 n-1}{12}\right) \Gamma\left(\frac{3 n+19}{12}\right) \Gamma\left(\frac{n+5}{4}\right)}{8 \sqrt{\pi}(n+1) \Gamma\left(\frac{n+7}{4}\right)}, a(5.52)=0.102 .
\end{aligned}
$$

We can relate this to the observed flux density, $S\left(\nu_{o}\right)$, in the optically thin region of the spectrum (43 GHz) using the relativistic transformation relations from Lind and Blandford $(1985)$,

$$
S\left(\nu_{o}\right)=\frac{\delta^{(3+\alpha)}}{4 \pi D_{L}^{2}} \int j_{\nu}^{\prime} d V^{\prime}
$$

where $D_{L}$ is the luminosity distance and $j_{\nu}^{\prime}$ is evaluated in the plasma rest frame at the observed frequency. A second equation arises from our SSA fit to the data above,

$$
\mu(\nu=19.5 \mathrm{GHz}) R=1 .
$$

Since, we already determined $\alpha=2.26$ then the pair of equations (12) and (13) with the expansions of equations $(7),(8),(10)$ and (6.7) indicate that we have two equations in four unknowns, $R, B, \delta$ and $N_{\Gamma}$. We explore this solution space by setting $R$ and $\delta$ fixed then solving (12) and (13) for $B$ and $N_{\Gamma}$, numerically. The results are displayed graphically in Figure 10. We solve for the actual number density, $N$, from the coefficient $N_{\Gamma}$ by integrating the powerlaw distribution for $\Gamma=5.52$ down to $\gamma=1, N \approx N_{\Gamma} / 4.22$. We justify the use of $\gamma=1$ as a minimum of integration in our discussion of Table 3 and equation (18) below. We randomly chose four values of $\delta$ that seem reasonable from VLBI studies of radio loud quasars, $\delta=1.00$ (non-relativistic), $\delta=2.10$ (mildly relativistic), $\delta=6.40$ and $\delta=9.40$ (highly relativistic) (Kellermann et al 2004; Vermeulen and Cohen 1994). For each value of $\delta$ we found an exact powerlaw solution for both $B$ and $N$. The powerlaw for $N$ is extremely steep. It is apparent that $N$ and $B$ take on extraordinary values outside of a narrow range of radii. These radii are smaller than the $43 \mathrm{GHz}$ beam minor axis - roughly between about $1 / 3$ to $1 / 30$ of the $43 \mathrm{GHz}$ beam minor axis, so the emission region can not be resolved by VLBA. 


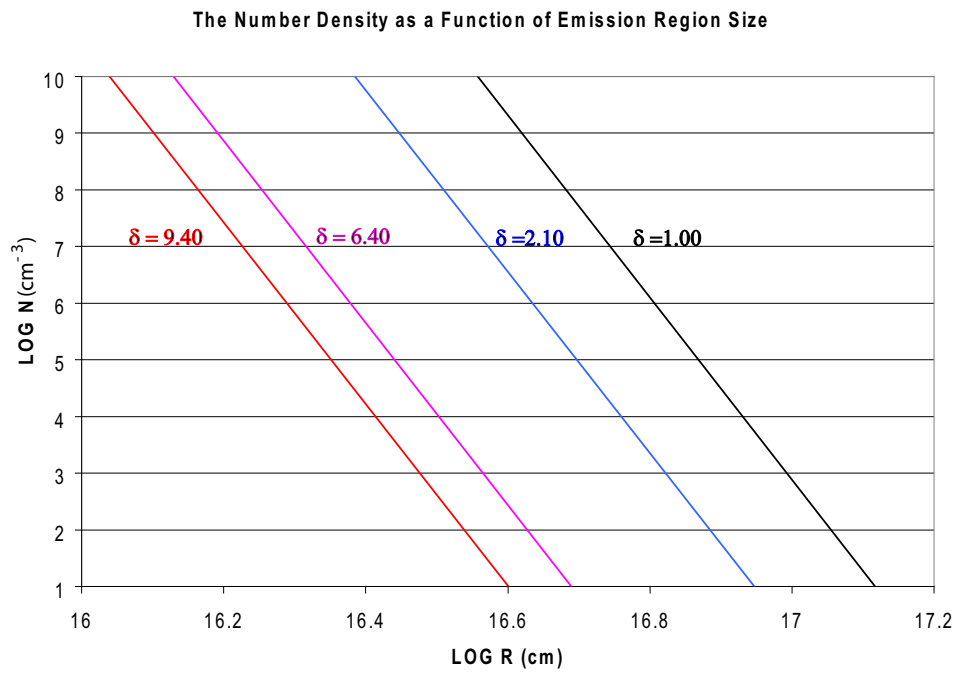

The Magnetic Field Strength as a Function of the Size of the Emission Region

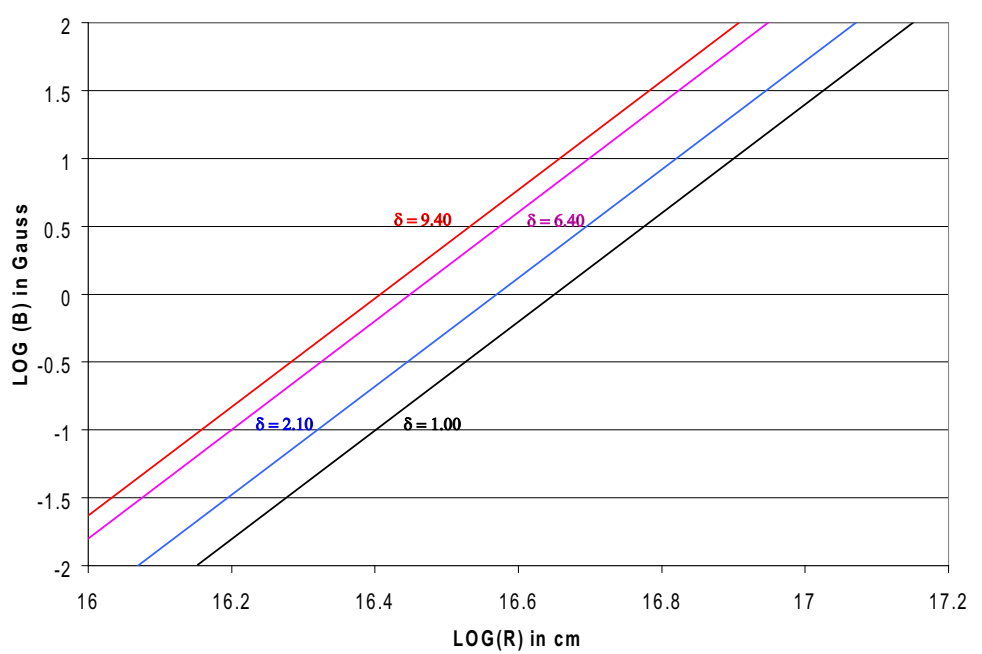

Fig. 10.- The number density (top) and the magnetic field strength (bottom) in a homogeneous synchrotron self-absorbed spherical model of the $22 \mathrm{GHz}$ flare at epoch 2006.32 as a function of Doppler factor, $\delta$ and spherical radius of the emission region, $\mathrm{R}$. 


\section{Minimum Jet Kinetic Luminosity}

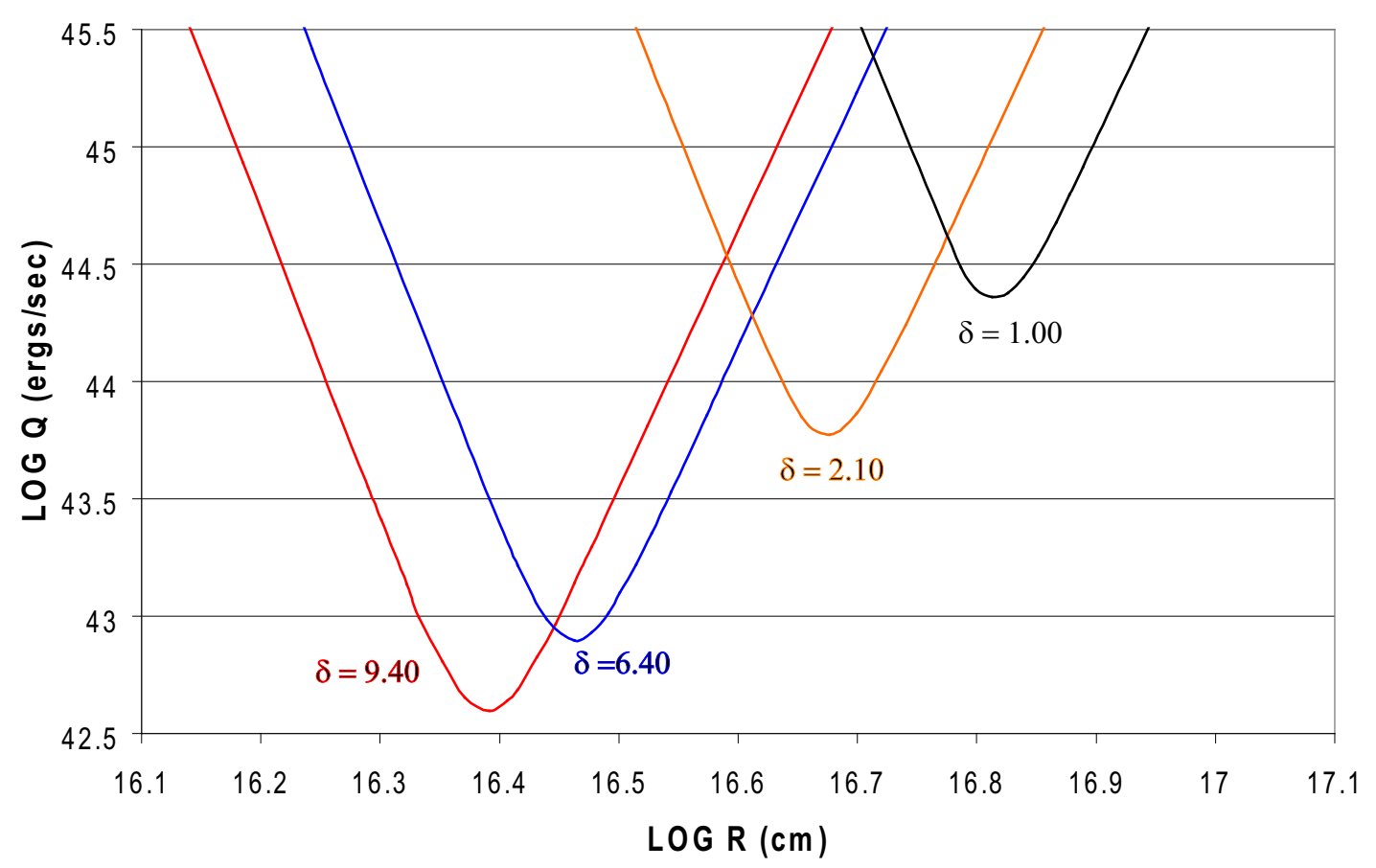

Fig. 11.- The minimum kinetic energy flux, $Q$, for homogeneous spherical source models as a function of $R$ and Doppler factor, $\delta$. 
It is straightforward to compute the total energy in a spherical volume from these solutions for $B$ and $N$,

$$
E(R)=\int\left(U_{B}+U_{e}\right) d V=\frac{4}{3} \pi R^{3}\left[\frac{B^{2}}{8 \pi}+\int_{1}\left(m_{e} c^{2}\right)\left(N_{\Gamma} \gamma^{-n+1}\right) d \gamma\right]
$$

The justification for using 1 as the minimum value of $\gamma$ in (14) is given in our discussion of Table 3 below. The energy in the observers frame is $E_{o}=\delta E$. From equation (14), we can derive a lower bound on the flare kinetic energy flux, $Q_{\min }(R)=\delta E(R) / t_{\max }$, where $t_{\max }=$ 91 days is the maximum amount of time that it took the flare to grow (i.e., the separation in time between our two observations in 2006). We plot the resulting $Q_{\min }(R)$ values in Figure 11 for each of the four values of $\delta$. Each of the four plots of $Q_{\min }(R)$ has a minimum that we designate as the minimum kinetic energy flux for that value of delta, $Q_{\min }$. To the left (right) of the minimum in each curve, the flow is thermally (magnetically) dominated. The minimum values follow an exact functional dependence on $\delta, \log Q_{\min }=44.36-1.8153 \delta$. The properties of these four $Q_{\min }$ solutions are tabulated in Table 3. The first column in Table 3 is the value of $\delta$ in the model. The next column is the minimum kinetic energy flux of the homogeneous spherical model of the flare. The next 3 columns are $\mathrm{R}, \mathrm{B}$ and $\mathrm{N}$ for that $Q_{\min }$ solution. The synchrotron lifetime measured in years in the observers frame is calculated in column 6 using the formula from Tucker (1975),

$$
t_{\text {syn }} \approx(\delta)^{-1} 5 \times 10^{11} B^{-1.5}\left(\nu_{o} / \delta\right)^{-0.5} \mathrm{sec} .
$$

We compute this at $22 \mathrm{GHz}$ in the observer's frame in order to see if the very steep spectrum from $22 \mathrm{GHz}$ to $43 \mathrm{GHz}$ in Table 1 can be explained by synchrotron cooling - spectral ageing. All of these lifetimes are short compared to 0.25 years between observations and since the flare probably expanded from smaller dimensions, $B$ was probably significantly larger in early stages of the flare, so the synchrotron cooling rate was probably larger than indicated by column 6 in the early stages of the flare evolution. Thus, we conclude that synchrotron cooling is a viable explanation of the very steep spectrum, high frequency tail of the core spectrum in Figure 5.

The next column tabulates the brightness temperature, $T_{b}$, if the core flux came from the spherical volume of radius, R, using the definition from Scheuer and Williams (1968)

$$
T_{b}=\frac{S_{\nu}}{\Omega} \frac{\mathrm{c}^{2}}{2 \mathrm{k}_{b} \nu^{2}} .
$$

The values approach the inverse Compton limit that is given in Marscher et al (1979) as (see also equations (19) and (20), below)

$$
T_{\max } \sim 10^{12} \delta^{1.2} \mathrm{~K} .
$$


The large $T_{b}$ values in Table 3 indicate that significant cooling of the relativistic electrons from inverse self-Compton losses are working in consort with synchrotron cooling to create the steep spectrum (Kellermann \& Pauliny-Toth 1969; Readhead 1994).

The last column in Table 3 is an estimate of electron energy $\gamma$ at the peak frequency. We know that the peak is less than $22 \mathrm{GHz}$, so these values are an upper bound. Using the formula form Tucker (1975), we compute the electron energy at $22 \mathrm{GHz}$ to form an upper bound,

$$
\gamma_{\text {peak }} \approx \frac{\nu_{o}(\text { peak }) / \delta}{\left(3 \times 10^{6} B\right)^{0.5}} .
$$

These are very low values for a relativistic jet and suggest that the energy spectrum likely goes down to $\gamma=1$ (i.e., there are electrons that radiate as low as $20 \mathrm{MHz}$, but the emission is not seen due to SSA). The low $\gamma$ values are related to the fact that all these minimum kinetic energy flux solutions are very highly magnetized.

The most revealing entry in Table 3 is the high value of $T_{b}$. The ratio of inverse Compton luminosity, $L_{i c}$, to synchrotron Luminosity, $L_{\text {synch }}$ was calculated in Readhead (1994) as

$$
\begin{aligned}
& \frac{L_{i c}}{L_{\text {synch }}}=\left(\frac{T_{b} G(\alpha, z)^{1 / 5}}{10^{12.22}}\right)^{5}\left[1+\left(\frac{T_{b} G(\alpha, z)^{1 / 5}}{10^{12.22}}\right)^{5}\right], \\
& G(\alpha, z)= \\
& {\left[\frac{v_{\mathrm{op}} / \delta}{3.5 \mathrm{GHz}}+\frac{v_{\mathrm{op}} / \delta}{(1+z) 1 \mathrm{GHz}} \times\left[\left(\frac{v_{\mathrm{high}}}{(1+z) v_{\mathrm{op}}}\right)^{(1+\alpha)}-1\right]\right] f_{3}(\alpha)^{-4}(1+z)^{4},}
\end{aligned}
$$

where $v_{\mathrm{op}}=19.5 \mathrm{GHz}$ is the peak in the observed SSA spectrum, $v_{\text {high }}=43 \mathrm{GHz}$ is the highest frequency in the power law in the observers frame (typically taken as $100 \mathrm{GHz}$ in the rest frame of the plasma). Our choice of $43 \mathrm{GHz}$ is conservative and $L_{i c} / L_{\text {synch }}$ might be slightly underestimated by this assumption. This expression also requires the coefficient, $f_{3}(\alpha)$, that is given by equation (15c) of Scheuer and Williams (1968). For the minimum kinetic energy flux solution with $\delta=1$ in Table 3, the data in Table 1 and equations (19) (20) imply that $L_{i c} / L_{\text {synch }} \approx 2 \times 10^{8}$. The X-ray observations from $0.2 \mathrm{keV}-50 \mathrm{keV}$ from Braito et al (2004) that indicate a total intrinsic X-ray luminosity of $L_{X} \sim 10^{44} \mathrm{ergs} / \mathrm{s}$ from the sum of accretion disk corona luminosity and the jet luminosity. Combining this with our measured radio luminosity of the core in Table 1 , we observe that

$$
\frac{L_{i c}}{L_{\text {synch }}} \sim \frac{10^{44} \mathrm{ergs} / \mathrm{s}}{10^{41} \mathrm{ergs} / \mathrm{s}}=10^{3} .
$$

Thus, the $\delta=1$ minimum kinetic energy flux solution is inconsistent with the value of $L_{i c} / L_{\text {synch }}$ determined by observation. 
One can investigate other possibilities in the $\delta=1$ solution space to see if a solution that is compatible with equation (21) is viable. Essentially, $R$ must be increased in order to lower $T_{b}$. Increasing the spherical radius of the $\delta=1$ solution so that $T_{b}$ satisfies equation (21) requires that $R>1.2 \times 10^{12} \mathrm{~cm}$. According to Figure 11, this would correspond to $Q \sim 10^{46} \mathrm{ergs} / \mathrm{s}$, the power of the strongest known FR II radio jets (Willott et al. 1999). These seems unlikely for a radio quiet quasar.

Alternatively, if we look at the $\delta=9.40$ solution at $R=3.12 \times 10^{16} \mathrm{~cm}$ we get an intrinsic value of $T_{b}^{\prime}=T_{b} / \delta=3.7 \times 10^{11} \mathrm{~K}$ (Readhead 1994). Inserting this into equations (19) and (20), implies that $L_{i c} / L_{\text {synch }} \lesssim 10^{3}$ for this solution in agreement with the bound set by observation in equation (21). From Figure 11, this solution is magnetically dominated and $Q \sim 3.5 \times 10^{43} \mathrm{ergs} / \mathrm{s}$. This value of $Q$ seems like a more reasonable value for a burst in a radio quiet quasars, on the order of the long term time averaged $Q$ in a strong $\mathrm{FR}$ I radio galaxy (Willott et al. 1999).

In summary, we argue that the $\delta=1.00$ solution is not physically reasonable on 2 counts:

- $T_{b}$ is right below the Compton limit for the minimum kinetic energy flux solution. If this were true then there should be a strong intrinsic X-ray source (and it should diminish the radio emissivity as well) (Kellermann \& Pauliny-Toth 1969). Extensive deep observations and very hard X-ray observations indicate that the intrinsic X-ray luminosity, $L_{X} \approx 1 \times 10^{44} \mathrm{ergs} / \mathrm{s}$ (Braito et al 2004). This is actually at the low end for the intrinsic X-ray luminosity distribution from the corona of the accretion disk of a quasar which has the thermal luminosity of Mrk 231 that was noted in the introduction, $L_{b o l} \gtrsim \times 10^{46} \mathrm{ergs} / \mathrm{s}$ (Laor et al 1997). Thus, the strong intrinsic radio emission and the relatively weak intrinsic X-ray emission (by radio quiet quasar standards) is not consistent with $T_{b}$ near the Compton limit. Alternatively, $R$ can be enlarged, but this presents different concerns as noted in the second point.

- The minimum flare kinetic energy flux is $Q_{\min }=2.30 \times 10^{44} \mathrm{ergs} / \mathrm{s}$. This is an Fanaroff Riley II (FR II) kinetic energy flux. The maximum FR I kinetic energy flux cutoffs at about $Q=5 \times 10^{43} \mathrm{ergs} / \mathrm{s}$ (Willott et al. 1999). Also notice that the kinetic luminosity rises very quickly away from the minimum in Figure 11, so this is a very loose lower bound on $Q$. In order to alleviate the first concern on $T_{B}$, above, a significant departure from the minimum kinetic energy flux solution is in order as discussed in regards to equation (21) and therefore a marked increase in the flare kinetic energy flux. It seems unlikely (but not impossible) that a radio quiet quasar should have flares with a strong FR II kinetic energy flux. 
Based on these two considerations, it seems difficult to reconcile the large kinetic energy flux with a Doppler factor of order unity in Mrk 231. Table 3 and the discussion of equations (19) - (21) indicates that a significant Doppler factor would ameliorate these concerns. It would also explain the gap between the core and the secondary in Figures 3 and 4 . Small deviations in the line of sight can greatly alter the flux density since by equation (12), $S_{\nu} \sim \delta^{5.26}$. Thus, small changes in the velocity or line of sight (jet wobble) can cause the jet emission between the core and secondary to be of such low surface brightness that it is too faint to be detected with VLBI. Similarly, a strong dependence on $\delta$ is compatible with the extreme flares and spectral variations that appear in Table 1. It seems likely that the subparsec scale radio jet in Mrk 231 is highly Doppler enhanced, i.e. relativistic and viewed pole-on. This is not unexpected since numerous BALQSOs have been determined to be viewed in a pole-on orientation based on radio variability $T_{b}$ values above the Compton limit (Zhou et al 2006; Ghosh and Punsly 2007). In particular, LoBALQSOs (those BALQSOs that exhibit low ionization resonant absorption troughs) seem inordinately common in these polar samples (1/3 - 1/2 of the polar BALQSOs). Mrk 231 is a LoBALQSO.

There is strong corroboration in the literature for our deduction that Mrk 231 hosts a polar relativistic jet. A $134 \pm 38 \mathrm{mJy}$ flux density variation in 1 day at $22.2 \mathrm{GHz}$ was reported in McCutcheon and Gregory (1978). Using the methods of Ghosh and Punsly (2007), the time variablity brightness temperature is $T_{b}=(12.4 \pm 3.5) \times 10^{12} \mathrm{~K}$. In order to avoid the inverse Compton catastrophe, the analysis in Ghosh and Punsly (2007) indicates that the line of sight to the jet is less than $\theta_{\max }=\left(25.6^{\circ}\right)_{-2.2^{\circ}}^{+3.2^{\circ}}$.

We also note the fortuitous circumstance that $8.33 \mathrm{GHz}$ VLBA observations were performed 0.35 years after the strong flare. epoch 2006.67 (BA80B). The historic high levels of $8 \mathrm{GHz}$ of core flux density at 2006.67 indicated in table 1 and figure 5 is supportive of the flare evolving to lower frequency 0.35 years later. Unfortunately we could not recover the 15 $\mathrm{GHz}$ data at 2006.67 which would have told us much more.

\section{The Nature of the Secondary Component}

In this section we study the interaction of the secondary component with the surrounding medium. It was shown in Ulvestad et al (1999a), using low frequency $(1.4 \mathrm{GHz}-15.3 \mathrm{GHz})$ VLBA observations that the compact radio double is seen through a FFA shroud. The FFA absorption creates a spectral turnover near $6 \mathrm{GHz}$. From Table 1, it is clear that the radio emission of the compact double is dominated by the secondary at low frequency. Thus, the

emission measure (see equation (6), $E M=\int N_{e}^{2} d l$ ), that is calculated in Ulvestad et al (1999a) from this $6 \mathrm{GHz}$ peak in the spectrum represents the shroud of gas that envelopes 
the secondary and might not be indicative of the gas density surrounding the core. In Ulvestad et al (1999a), it was stated that $E M \approx 1.2 \times 10^{8} \mathrm{~cm}^{-6} \mathrm{pc}$ produced the spectral turnover. However, it was not clear what temperature was assumed for the gas. Thus, we re-derive this result in a form that incorporates the temperature.

In order to study the spectral turnover, we want to use the lowest frequencies possible in order define the shape of the spectral peak accurately. The compact parsec scale double discussed thus far in this paper appears as an unresolved "core" at low frequency. The "core" is part of a triple radio source at low frequency. Components to the north and south of the core are detected at $8.4 \mathrm{GHz}$ and below. At $2.3 \mathrm{GHz}$, the resolution is not sufficient to avoid confusion between the "core" flux and flux from the north/south extended components. Thus, as a compromise, we chose data at a resolution matched to the $5 \mathrm{GHz}$ observation. We fit the data using equation (5) and a background spectral index of $\alpha=1.7$ for the synchrotron source. Our best fit used a covering factor, $f=0.98$. The corresponding solution for the emission measure is close to the value stated in Ulvestad et al (1999a),

$$
E M \approx 9 \times 10^{7} \mathrm{~cm}^{-6} \mathrm{pc}\left(\frac{T}{10^{4} \mathrm{~K}}\right)^{1.5}, 2 \times 10^{3} \mathrm{~K}<T<5 \times 10^{4} \mathrm{~K} .
$$

Considering that the secondary is surrounded by a dense cloud of free electrons as indicated in equation (22) and the secondary does not move appreciably according to Table 2 , even though the core jet is relativistic, it is natural to consider the possibility that the secondary is being braked to a slow speed by the surrounding gas that supports $N_{e}$. In physical language, is the pressure of the secondary in dynamic equilibrium with the ram pressure of the surrounding gas? To explore this possibility we first try to estimate the pressure within the secondary.

To estimate the pressure, we first find the $Q$ associated with the secondary and use the physical size to convert it to a pressure. Since the secondary is very steep spectrum at all epochs and is mildly variable, we consider it to be a compact analog of the radio lobes that are seen in classical double radio sources 4 . Furthermore, this compact lobe is seen through the effects of a FFA shroud and we can not detect its low frequency emission, even though

\footnotetext{
${ }^{4}$ One might also think of this feature as a strong knot in a jet that disappears, then reappears as a diffuse low frequency large scale elongated structure (that is depicted by the low frequency VLBA observations in Ulvestad et al (1999a)) that is barely detected with $8.4 \mathrm{GHz}$ VLBA at a PA $65^{\circ}$ offset from the sub-pc scale jet direction. The very steep spectral index in figure 6 , above $8.4 \mathrm{GHz}$ (which equates to a background spectral index of $\alpha=1.7$ in the FFA fit above), seems more indicative of lobe emission than knot emission. For such a steep spectrum structure, the distinction of knot or lobe is probably irrelevant with respect to the application of estimate of the jet kinetic energy flux in equation (23)
} 
it is plausibly $>1$ Jy considering the $8.4 \mathrm{GHz}$ flux density in Table 1 . Perhaps the most sophisticated estimate of jet kinetic energy flux was developed by Willott et al. (1999) which incorporates deviations from the overly simplified minimum energy estimates. The method also considers geometric effects, filling factors, protonic contributions and low frequency cutoff. The long term time average of the jet kinetic energy flux of a steep spectrum radio lobe, $\bar{Q}$, is estimated in Willott et al. (1999):

$$
\bar{Q} \approx 3.8 \times 10^{45} L_{151}^{6 / 7} \mathrm{ergs} / \mathrm{s}
$$

where $L_{151}$ is the total optically thin luminosity from the lobes in units of $10^{35} \mathrm{ergs} / \mathrm{Hz} / \mathrm{sr}$ at $151 \mathrm{MHz}$. The obstacle to implementing equation (23) is that it depends on the $151 \mathrm{MHz}$ flux density. This is problematic since the $151 \mathrm{MHz}$ flux from the secondary is blocked from our view by the FFA shroud. The lowest frequency measurement that we have in Table 1 is 8.4 GHz and we need to extrapolate this value to $151 \mathrm{MHz}$. We know that the spectrum is very steep between $8.4 \mathrm{GHz}$ and $15 \mathrm{GHz}, \alpha=1.38$, even with some diminished flux at 8.4 $\mathrm{GHz}$ from FFA. There are very few (if any) known radio lobes that are this steep between $151 \mathrm{MHz}$ and $8.4 \mathrm{GHz}$. Thus, there is no justification for extrapolating the $8.4 \mathrm{GHz}$ flux density with $\alpha=1.38$. A more typical value for steep lobe emission seems to be $\alpha \approx 0.8$ (Kellermann et al 1969; Herbig and Readhead 1992). Under this assumption, we used Table 1 and equation (23) to estimate the long term time average kinetic energy flux of the putative jet connecting the core to the secondary, $\bar{Q} \approx 9.9 \times 10^{41} \mathrm{erg} / \mathrm{s}$.

Even though this estimate of $\bar{Q}$ is crude, we can use it to estimate the pressure in the secondary. We can approximate the energy flux through the jet as $\bar{Q} \approx(1 / 4) \pi D^{2} v_{a d v} U$, where $\mathrm{D}$ is the diameter of the lobe, $v_{a d v}$ is the velocity that the lobe is advancing into the external medium and $U=U_{e}+U_{B}$ is the total internal energy density. Since in a relativistic gas the pressure $P_{\text {lobe }}=(1 / 3) U$, we estimate

$$
P_{l o b e} \approx \frac{4 \bar{Q}}{3 \pi D^{2} v_{a d v}}
$$

If the lobe is confined by the ram pressure of an external medium moving with a velocity, $v_{\text {ext }}$, and density, $m_{p} N_{\text {ext }}$, then we compute the pressure balance at the leading edge of the lobe to find

$$
P_{l o b e} \approx \frac{4 \bar{Q}}{3 \pi D^{2} v_{a d v}} \approx m_{p} N_{e x t}\left(v_{a d v}-v_{e x t}\right)^{2} .
$$

If we implement a fiducial distance of $1 \mathrm{pc}$ and assume that the medium is fully ionized then by equation $(22), N_{\text {ext }} \approx 10^{4} \mathrm{~cm}^{-3}$. We also get a value of $D=7.70 \times 10^{17} \mathrm{~cm}$ from the angular size of the emission region, 0.31 mas (this was determined from model fits to 
the $15 \mathrm{GHz}$ VLBA data by Ulvestad et al (1999b) and was verified by our fits in various epochs as well). We can then solve (25) with these parameters derived from the FFA fit and the VLBA data to get a qualitative understanding of the ram pressure confinement of the lobe. We explore equation (25) in two limits, first the enveloping gas is stagnant $v_{\text {ext }}=0$ which yields $v_{a d v}=0.012 c=3490 \mathrm{~km} / \mathrm{sec}$. Secondly, we note that the distance from the secondary to the core is typical for the location of the LoBAL gas. The velocity of the MgII absorption trough relative to the $\mathrm{H} \alpha$ emission (which we take as an approximation to the quasar rest frame) from Smith et al (1995) can be equated with the velocity of the enveloping medium, $v_{\text {ext }} \approx 0.0173 c$, from which equation $(25)$ implies that $v_{a d v}=0.025 c=$ $7570 \mathrm{~km} / \mathrm{sec}$. These values are consistent with the slow advance speed indicated in Table 2. The dynamic estimates correspond to a separation increase between the core and the secondary in 9.4 years of $0.041 \sin \theta$ mas and $0.089 \sin \theta$ mas, respectively (where $\theta$ is the angle between the line of sight and the jet propagation axis). These values are consistent with our observations which by equation (4) bounds $v_{a d v}<(0.053 / \sin \theta) c$, at the one sigma level.

In summary, the relativistic jet is over-pressurized relative to the gas density of the FFA absorbing gas, so it advances. However, the FFA gas is very dense and the kinetic energy flux is modest, thus the jet can drill through the dense medium relatively slowly at a non-relativistic rate. Independent of the assumptions involved in deriving and evaluating equation (25), this qualitative conclusion is robust and is consistent with the observed VLBA component separation data in Table 2.

This analysis raises the question, what is the medium that is confining the relativistic jet and braking its velocity to a virtual halt? Is it the putative dusty molecular torus, $v_{\text {ext }}=0$, or is it the BAL gas, $v_{e x t}=v_{B A L}$ ? We argue that the FFA absorbing screen is more likely the BAL gas than the molecular torus based on the following points.

- The low frequency VLBI observations of Lonsdale et al (2003) led them to conclude that "the lack of $21 \mathrm{~cm}$ absorption or maser amplification against the compact core (which is the compact double with our higher resolution) shows that the molecular disk does not cover the central source."

- The high Doppler factor inferred from our modeling of the core flare indicates that the jet is viewed in a nearly pole-on orientation. Furthermore, the existence of BAL troughs in the spectrum of Mrk 231 indicates that our line of sight is through the BAL gas. Thus, the jet direction seems pass through the BAL gas on pc scales.

In summary, the observational data is consistent with the jet ramming through the BAL gas and inconsistent with the jet plowing through the molecular disk. Thus, we proceed to 
consider the BAL gas as the FFA medium.

If the jet is ramming through the BAL gas then the jet can be used as a remote probe of properties of the BAL region. Thus, MRK 231 might be uniquely suited for studying the BAL gas. We find that the properties of the gas derived in this section are consistent with published interpretations of BALQSO physics. The main piece of information is the emission measure in equation (22), that does not have a unique decomposition in terms of the number density distribution $N_{e}(r)$ and the extent of the FFA region, $L$. In Ulvestad et al (1999a), they chose $L=1 \mathrm{pc}$ and a constant $N_{e}=1.1 \times 10^{4} \mathrm{~cm}^{-3}$. We interpret this value of $L$ within the context of the polar models of BAL outflows flows in Punsly (1999a, b ${ }^{2}$ ); Proga and Kallman (2004). Consider a conical outflow that experiences very little acceleration as might be the case in the LoBAL region (near UV lines) since most of the line driving acceleration is provided by far UV and EUV absorption lines (Murray et al 1995). In this scenario, by conservation of mass, $N_{e}(r)=N_{o} / r^{2}$ and we can integrate the emission measure in general form as

$$
E M=\int_{L}^{\infty} N_{o}^{2} r^{-4} d r=\frac{1}{3}\left(N_{e}(r=L)\right)^{2} L, L=L_{o b s} / \sin \theta,
$$

where the observed $L_{o b s}$ is the projection of $L$ in the sky plane and $\theta$ is the angle to the line of sight to the radio jet axis (symmetry axis of the BAL outflow). In Punsly (1999b), it was calculated that $\theta=15^{\circ}$ was typical for a LoBAL or from Table $2, L=3.75$ pc and from equations $(22)$ and $(25), N_{e}(r=L) \approx 4.9 \times 10^{3} \mathrm{~cm}^{-3}\left(\frac{T}{10^{4} \mathrm{~K}}\right)^{0.75}$. If we assume that the absorbing column is almost completely ionized $\left(N_{e} \approx N\right)$ in the BAL region, as deduced in Hamann (1998), then we can estimate the total hydrogen column,

$$
N_{H}=\int_{L}^{\infty} N(r) d r \approx 5.6 \times 10^{22}\left(\frac{T}{10^{4 \circ} \mathrm{K}}\right)^{0.75} \mathrm{~cm}^{-2} .
$$

The total hydrogen column is sufficient to make the BAL absorption lines optically thick to resonant absorption (Murray et al 1995; Hamann 1998). Superficially, one would think that the value of $N_{H}$ in equation (27) is incompatible with the BAL gas in Mrk 231. This is actually not the case as discussed at length in Hamann (1998); Hamann et al (2003). It was found that many BALQSOs show absorption in PV $\lambda \lambda 1118,1128$ with an optical depth $\geq 0.2$. For such a rare species this is impossible, unless the CIV $\lambda \lambda 1548,1550$ troughs are severely saturated. After studying many high resolution spectra of BALQSOs, Hamann et al (2003) concluded that over half of the BALQSOs with deep far UV spectra showed significant PV $\lambda \lambda 1118,1128$ absorption. The implication was that these BALQSOs must have CIV $\lambda \lambda 1548,1550$ troughs that are completely saturated with the bottoms of the troughs filled in by scattered light and un-attenuated (or mildly attenuated) lines of sight that are a consequence of partial covering of the central AGN by the predominant thick absorber. 
After, numerically modeling the absorption region, Hamann (1998) found that the CIV $\lambda \lambda 15481550$ troughs are saturated as a consequence of absorption presented by a column that contained almost completely ionized hydrogen, $10^{22} \mathrm{~cm}^{-2}<N_{H}<10^{24} \mathrm{~cm}^{-2}$. The upper limit is set by the fact that the gas must be optically thin to Compton scattering. It was concluded that these large column densities are likely common in BAL regions. Based on the analysis of Hamann (1998); Hamann et al (2003), the values of $N_{H}$ determined by equation (27) are typical of many BALQSO absorbing columns. Furthermore, we remind the reader that there is very little neutral hydrogen absorption towards the nuclear secondary as noted in Lonsdale et al (2003). Yet by equations (22) and (27) there are large values of EM and $N_{H}$ which implies that the intervening hydrogen column density must be almost completely ionized as expected from the ionization models of dense BAL winds in Hamann (1998). In particular, the photo-ionization models of Hamann (1998) yield a range of allowed ionization states: for a total hydrogen column density of $N_{H}=6 \times 10^{22} \mathrm{~cm}^{-2}$, the neutral hydrogen column density is quite small, $5 \times 10^{16} \mathrm{~cm}^{-2}<N_{H I}<5 \times 10^{17} \mathrm{~cm}^{-2}$.

From our analysis above, we have enough parameters to estimate the kinetic energy flux of the the BAL wind, $Q(B A L)$ in terms of the parameters of the enveloping gas surrounding the leading edge of the secondary. First, assume that the BAL wind has a bulk velocity in the range of velocity spread given by the MgII BAL absorbing gas and NaI D absorbing gas relative to the quasar rest frame, $0.0151 c<v_{B A L}<0.0173 c$. From the numerical models in Punsly (1999b), a polar LoBAL wind will have an opening angle of $\approx 20^{\circ}-30^{\circ}$. Combining this opening angle and the BAL wind velocity with the 3.75 pc displacement from the nucleus and $N_{e} \approx 4.9 \times 10^{3} \mathrm{~cm}^{-3}$ yields $2 \times 10^{43} \mathrm{ergs} / \mathrm{s}<Q(B A L)<6 \times 10^{43} \mathrm{ergs} / \mathrm{s}$. It is curious that this is similar to the kinetic energy flux of the relativistic flare that was discussed in section 5 . One can compare this parametric analysis, with the theoretical polar BAL models in Table 1 of Punsly (1999b). First of all, for the numerical models, the kinetic energy flux in the BAL wind solutions is $7.2 \times 10^{42} \mathrm{ergs} / \mathrm{s}<Q_{\text {theoretical }}(B A L)<2.3 \times 10^{44} \mathrm{ergs} / \mathrm{s}$, in agreement with what we deduced for MRK 231. However, the mass density in the BAL wind of Mrk 231 is estimated to be a factor of 10 - 50 times larger than that which is predicted by mass conservation in the numerical models. A possible explanation of the additional gas density is that the polar BAL entrains gas as it propagates through the surrounding medium. This seems reasonable on two counts. First of all, there is the dusty, enveloping gas that partially obscures the nuclear region and reddens the spectrum. This gas distribution could be a consequence of the merger that is in progress with a nearby smaller galaxy to the south (Armus et al 1994). So there is a potential for significant gas entrainment. Secondly, the BAL absorption is deep, but not particularly blue-shifted for a BALQSO, only about 5000 $\mathrm{km} / \mathrm{s}$ : this is consistent with a resistive drag resulting from mass loading of the wind. 


\section{Discussion}

In this article, we used high frequency VLBA observations to determine that the central engine of the nearby BALQSO Mrk 231, ejects relativistic plasma along a trajectory close to the line of sight. Furthermore, it appears that this jet is interacting strongly with the dense BAL gas at a de-projected distance $\sim 3-4$ pc from the radio core.

The most striking finding was the strong $22 \mathrm{GHz}$ flare that emerged from the core between epochs 2006.07 and 2006.32. The core spectrum, during the flare, is characterized by extreme gradients, namely it transitions from being very steep spectrum, $\alpha \approx 2$ above $22 \mathrm{GHz}$ to being inverted $\alpha<0$ at $\approx 15 \mathrm{GHz}$. The magnitude of the flare combined with the three month interval between observations sets a lower bound on the time variability brightness temperature of $T_{b}>2 \times 10^{10} \mathrm{~K}$, below the inverse Compton limit, $\sim 10^{12} \mathrm{~K}$. However, all attempts in section 5 to model the high frequency peak of the spectral turnover, $19.5 \mathrm{GHz}$, in combination with the steep spectral index above $22 \mathrm{GHz}(\alpha \approx 2)$ indicate that the flare is synchrotron self-absorbed and $T_{b} \approx 10^{12} \mathrm{~K}$, unless the flux density is Doppler boosted. Our Doppler boosted models indicate a kinetic energy flux, $Q \sim 3 \times 10^{43} \mathrm{ergs} / \mathrm{sec}$ and an intrinsic (rest frame of the plasma) brightness temperature $\gtrsim 10^{11} \mathrm{~K}$.

Our discovery of a relativistic jet in a radio quiet quasar does not stand alone. There are the aforementioned BALQSOs in Ghosh and Punslv $(2007) ;$ Zhou et al (2006) as well as PG 1407+263 in Blundell et al (2003) and III Zw 2 in Brunthaler et al (2000). By definition, most radio quiet quasars are weak radio sources, so there are not many observations with VLBI. Consequently, we can not say if the relativistic jets that have been detected are more the exception or the rule.

The compact nature of the jet emission in MRK231 and the strong interaction with the surrounding medium has many strong similarities to $\mathrm{GHz}$ peaked (GPS) radio sources (O’Dea 1998). In the interval between 2006.07 and 2006.32, Mrk 231 seemed to transform from a weak version of a GPS source (as it was in 1996.93) to a weak version of a rare class of radio sources known as high frequency peakers, but on a scale 1000 times smaller (Orienti and Dallacasa 2008). It is weakly polarized like GPS sources, but unlike GPS sources Mrk 231 is highly variable (O'Dea 1998). We were unable to reliably detect any morphological changes associated with this change in character. It would be interesting to see if long term VLBA monitoring at $43 \mathrm{GHz}$ and $22 \mathrm{GHz}$ would reveal ejecta emanating from the core $\sim$ a month after a flare. Another interesting aspect of high frequency VLBA monitoring would be to understand the flare growth and decay time scales. The information gleaned from this data would further constrain the physical model of section 5 . This monitoring would undoubtedly reveal large time variability brightness temperatures and might confirm the presence of synchrotron cooling, i.e. is there a short lived $43 \mathrm{GHz}$ precursor to a $22 \mathrm{GHz}$ 
flare. If the basic SSA model of the flare that was presented in section 5 is confirmed by these observations, a natural question arises: what is the origin of the strong magnetic field , a few $\mathrm{G}$, on scales approaching $10^{17} \mathrm{~cm}$ ? If the field propagates from the base of the jet, does it tell us something about the turbulent plasma inside the accretion disk?

Another important finding was that the core was unpolarized even during a flare. This implies that the magnetic field is not ordered or there is a huge amount of weakly magnetized gas around the central engine which depolarizes the synchrotron emission by Faraday rotation. The arguments in section 5 , regarding $T_{b}$ seemed to favor magnetically dominated models. It is hard to understand how a strong field would not be ordered in a magnetically dominated plasma. This would seem to indicate a dense magnetized envelope surrounding the radio core. Perhaps this is the source of the seed field that is ultimately responsible for the strong magnetic field in the core flares.

It is intriguing that in spite of the highly relativistically beamed Doppler core that the steep spectrum secondary $(\alpha>1)$ is mildly variable and has very little apparent motion. This peculiar situation was studied in section 6 . A standard estimate of the long term time averaged kinetic energy flux of the secondary reveals $\bar{Q} \approx 10^{42} \mathrm{ergs} / \mathrm{sec}$ which is consistent with the core having episodic flares of $Q \gtrsim 10^{43} \mathrm{ergs} / \mathrm{sec}$, if the two components are part of the same jetted beam of plasma. Thus, we established consistency between the analysis of the core in section 5 and the kinetic energy flux of the secondary that was estimated in section 6. We also noted that matched (to $5 \mathrm{GHz}$ ) resolution VLBA observations indicates that the secondary is seen through a shroud of free-free absorbing gas with an emission measure of $\approx 10^{8} \mathrm{~cm}^{-6} \mathrm{pc}$. We argue that the steep spectrum secondary seems to be a radio lobe associated with the jet advancing into a dense medium (the jet is confined by ram pressure) that is also the source of the free-free absorption. The properties of the dense gas are consistent with a temperature, $10,000 \mathrm{~K}-20,000 \mathrm{~K}$, a displacement from the nucleus, 3$4 \mathrm{pc}$, and a total hydrogen column density, $N_{H}=10^{22}-10^{23} \mathrm{~cm}^{-2}$. These gas parameters are often associated with the BAL wind (Hamann et al 2003). The implied kinetic luminosity of this BAL wind is within the narrow range of values that are predicted by the polar BAL wind theory in Punsly (1999b). The density is at least an order of magnitude larger than what is expected from such a wind, indicating that there is substantial gas entrainment as the BAL wind propagates outward from the nucleus.

The jet in Mrk 231 might offer a unique opportunity to study the central dynamics of a radio quiet quasar. Not only is the quasar near to earth, but the jet is the brightest at high frequency of any radio quiet quasar jet. Thus, no other radio quiet quasar central engine can be explored with such high resolution (VLBI). Furthermore, now we know the orientation of the jet. The high resolution of $43 \mathrm{GHz}$ VLBA allows us to peer into the inner regions of this 
quasar, using the relativistic jet as a probe of the X-ray emitting gas, the X-ray absorbing gas, and the BAL wind. In summary, there are many potential discoveries that can be made with future VLBA monitoring coordinated with observations at other frequency bands. Our observations lead us to ask the the following questions

1. Is there more evidence of superluminal motion, like apparent velocities of radio emission larger than the speed of light that can be detected?

2. What is the maximum kinetic energy flux of the high frequency flares?

3. Are these types of flares present in other radio quiet quasars, but with varying magnitude?

4. Why are the flares so powerful, yet cool off so fast that MRK 231 never becomes a strong FR I radio source? More specifically, why do all the components in the the core and secondary seem to show such steep high frequency spectra? What is the powerful cooling mechanism?

5. Is this cooling mechanism found in all radio quiet quasars and why does it not occur in radio loud quasars?

6. Is the gas that entrains the secondary one and the same as the BAL gas?

7. How fast do strong flares grow and what are the details of their rapid decay?

8. Do the detected (but absorbed) X-rays come primarily from the same plasma that is responsible for the $22 \mathrm{GHz}$ flares (i.e., the accretion disk X-rays are completely attenuated)? Do we see correlated flares in the X-ray and microwave bands?

9. Since the microwave flare emission is from such small scales, it is nearly coincident with the scales of X-ray emission and absorption. Thus, can we expect a strong interplay between microwave flares and the X-ray spectral properties of MRK231?

10. If the X-rays in Mrk 231 are predominantly inverse Compton emission from the microwave jet, does the dense X-ray absorption column constrain the physical parameters of the enveloping medium that gets entrained by the propagating jet and thwarts it propagation to large scales?

11. Does a strong microwave flare blast holes in the X-ray absorbing column allowing us to get a brief mildly attenuated glimpse of the X-ray emission from the accretion disk corona? 
12. Do flares in the microwave correspond to a decrease in the X-ray emission of the accretion disk corona as has been hypothesized as a universal phenomenon in black hole accretion systems (the "low-hard" state)(Maccarone et al 2003; Marscher et al 2002)?

Based on these considerations that were raised by our observations, it seems clear that a coordinated observing program of VLBA and X-ray observations would shed light on many of these issues, in particular points 8 to 12. Generally speaking, coincident X-ray and VLBA monitoring is potentially a powerful method of studying any "disk-jet connection."

\section{REFERENCES}

Armus, L. et al 1994, AJ 10876

Beasley, A., Conway, J., ASP Conf. Ser. 82, Very Long Baseline Interferometry and the VLBA, ed. J. A. Zensus, P. J. Diamond \& P. J. Napier (San Francisco: ASP), 328

Becker. et al 2000, ApJ 53872

Blundell, K., Beasley, A., Bicknell, G. 2003, ApJL 591103

Bokensberg et al 1977, MNRAS 178451

Braito, V. et al 2004, A \& A 42079

Briggs, D., 1995, PhD Thesis, NRAO

Britzen, S. et al 2007, A \& A476 759

Brunthaler, A. et al 2000, A \& A 357 L45

Condon, J. et al 1998, AJ 1151693

Cotton, W. 1993, AJ 1061241

Falcke, H., Patnaik, A., Sherwood, W, 1996, ApJL 473 L13

Farrah, D et al 2003, MNRAS 343585

Ghosh, K. and Punsly, B. 2007 ApJL 661139

Ginzburg, V. and Syrovatskii, S. 1969, Annu. Rev. Astron. Astrophys. 7375 
Hamann, F. 1998 ApJ 500798

Hamann, F., Sabra, B., Junkkarinen, V. Cohen, R. and Shileds, G. 2003 in MPE Report Proceedings of Workshop; "X-ray Spectroscopy of AGN with Chandra and XMM-Newton" eds.,Th Boller, S. Komossa, S., Kahn, S. and Kunieda, H. arXiv:astro-ph/0304564

Herbig, T., Readhead, A. 1992, ApJS 8183

Homan, D. 2002, ApJ 56899

Kellerman, K.I. et al 1989, AJ 981195

Kellerman, K.I. et al 2004, ApJ 609539

Kellermann, K. I., \& Pauliny-Toth, I. I. K. 1969 ApJ, 155, L71

Kellermann, K. I., Pauliny-Toth, I. I. K., Williams, P. J. S. 1969 ApJ 1571

Konigl, A. 1981, ApJ 243700

Laor, A. et al 1997, ApJ 47793

Leppänen, K., 1993, VLBA Scientific Memo 1, http://www.vlba.nrao.edu/memos/sci

Leppänen, K., Zensus, J., Diamond, P., 1995, AJ 1102479

Lind, K., Blandford, R. 1985, ApJ 295358

Lipari, S., Colina, L., Macchetto, F. 1994, ApJ 427174

Lobanov, A. 1998, A \& A 33079

Lobanov, A. 2005 arXiv:astro-ph/0503225

Lonsdale, C. et al 2003, ApJ 592804

Maccarone, T., Gallo, E., Fender, R. 2003, MNRAS 345 L19

Marscher, a. et al 1979, ApJ 233498

Marscher, a. et al 2002, Nature 417625

Martel, A. 1997, PhD Thesis, Univ. California Santa Cruz

McCutcheon, W., Gregory, P. 1978 , AJ 83566 
Moffet, A. 1975 in Stars and Stellar Systems, IX: Galaxies and the Universe, eds. A. Sandage, M. Sandage \& J. Kristan (Chicago University Press, Chicago), 211.

Murray, N. et al 1995, ApJ 451498

O’Dea, C. 1998, PASP 110493

Orienti, M. and Dallacasa, D. 2008, A \& A 487885

Proga, Kallman, T. ApJ 2004616688

Punsly, B. 1999, ApJ 527609

Punsly, B. 1999, ApJ 527624

Punsly, B., Tingay, S. 2005, ApJL 633 L89

Readhead, A. 1994, ApJ 42651

Reynolds, C. S., Fabian, A., Celloti, A., Rees, M. 1996, MNRAS 283873

Savolainen, T., Wiik, K., Valtaoja, E., Tornikoski, M 2006, A \& A 44671

Scheuer, P, Williams, P. 1968, Annu. Rev. Astron. Astrophys. 6321

Shepherd, M., Pearson, T., Taylor, G. 1994, BAAS, 26, 987

Shepherd, M., Pearson, T., Taylor, G. 1994, BAAS, 27, 903

Smith, P. et al 1995, ApJ 444146

Telfer, R., Zheng, W., Kriss, G., Davidsen, A. 2002, ApJ 565773

Tucker, W. 1975, Radiation Processes in Astrophysics (MIT Press, Cambridge).

Ulvestad, J., Wrobel, J. and Carilli, C. 1999, ApJ516 134

Ulvestad, J. et al 1999, ApJL517 L81

Ulvestad, J., Antonucci, R., Barvainis, R. 2005, ApJ 621123

Ulvestad, J., VLBA Observational Status Summary, http://www.vlba.nrao.edu/astro/obstatus/current/

Vermeulen, R. Cohen, M. 1994, ApJ 430467

Walker, R. C., Dhawan, V., Romney, J., Kellermann, K., Vermeulen, R. 2000, ApJ 530233 
Weymann, R.J., Morris, S.L., Foltz, C.B., Hewett, P.C. 1991, ApJ 373, 23

Weymann, R. 1997 in ASP Conf. Ser. 128, Mass Ejection from Active Nuclei ed, N.Arav, I. Shlosman and R.J. Weymann (San Francisco: ASP) 3

Willott, C., Rawlings, S., Blundell, K., Lacy, M. 1999, MNRAS 3091017

Zheng, W. et al 1997, ApJ 475469

Zhou, H. et al 2006, ApJ 639716

This preprint was prepared with the AAS LATEX macros v5.2. 
Table 3: Details of the Minimum kinetic energy flux Solutions

\begin{tabular}{cccccccc}
\hline$\delta$ & $\begin{array}{c}Q_{\min } \\
\text { ergs } / \mathrm{s}\end{array}$ & $\begin{array}{c}\mathrm{R} \\
\mathrm{cm}\end{array}$ & $\begin{array}{c}\mathrm{B} \\
\text { Gauss }\end{array}$ & $\begin{array}{c}\mathrm{N} \\
\mathrm{cm}^{-3}\end{array}$ & $\begin{array}{c}t_{\text {syn }}=22 \mathrm{GHz} \\
\mathrm{yrs}^{\mathrm{a}}\end{array}$ & $\begin{array}{c}T_{b} / T_{\max } \\
\mathrm{b}\end{array}$ & $\begin{array}{c}\gamma \\
\text { peak } \\
\mathrm{c}\end{array}$ \\
\hline 1.00 & $2.29 \times 10^{44}$ & $6.50 \times 10^{16}$ & 4.43 & $3.35 \times 10^{6}$ & $1.15 \times 10^{-2}$ & 0.86 & $<40.7$ \\
2.10 & $5.94 \times 10^{43}$ & $4.75 \times 10^{16}$ & 2.69 & $8.80 \times 10^{5}$ & $1.68 \times 10^{-2}$ & 0.66 & $<36.1$ \\
6.40 & $7.86 \times 10^{42}$ & $2.90 \times 10^{16}$ & 1.14 & $1.81 \times 10^{5}$ & $3.48 \times 10^{-2}$ & 0.47 & $<31.7$ \\
9.40 & $3.94 \times 10^{42}$ & $2.47 \times 10^{16}$ & 0.86 & $9.75 \times 10^{4}$ & $4.38 \times 10^{-2}$ & 0.41 & $<30.1$
\end{tabular}

${ }^{a}$ measured in observers frame, see equation (15)

${ }^{b}$ See equations (16) and (17). Based on $68.6 \mathrm{mJy}$ at $15 \mathrm{GHz}$.

${ }^{c}$ see equation (18), $\nu_{o}($ peak $)<22 \mathrm{GHz}$ 